ARTICLE

\title{
Dysregulation of bile acids increases the risk for preterm birth in pregnant women
}

\author{
Sangmin You ${ }^{1,4}$, Ai-Min Cui ${ }^{2,4}$, Syed F. Hashmi ${ }^{1} 4$, Xinmu Zhang ${ }^{1}$, Christina Nadolny ${ }^{1}$, Yuan Chen ${ }^{1}$, Qiwen Chen ${ }^{1}$,
} Xin Bush ${ }^{1}$, Zachary Hurd ${ }^{1}$, Winifer Ali $^{1}$, Gang Qin $\mathbb{1}^{3}$ \& Ruitang Deng ${ }^{1 \times}$

Preterm birth (PTB) is the leading cause of perinatal mortality and newborn complications. Bile acids are recognized as signaling molecules regulating a myriad of cellular and metabolic activities but have not been etiologically linked to PTB. In this study, a hospital-based cohort study with 36,755 pregnant women is conducted. We find that serum total bile acid levels directly correlate with the PTB rates regardless of the characteristics of the subjects and etiologies of liver disorders. Consistent with the findings from pregnant women, PTB is successfully reproduced in mice with liver injuries and dysregulated bile acids. More importantly, bile acids dose-dependently induce PTB with minimal hepatotoxicity. Furthermore, restoring bile acid homeostasis by farnesoid $X$ receptor activation markedly reduces PTB and dramatically improves newborn survival rates. The findings thus establish an etiologic link between bile acids and PTB, and open an avenue for developing etiology-based therapies to prevent or delay PTB.

\footnotetext{
${ }^{1}$ Department of Biomedical and Pharmaceutical Sciences, College of Pharmacy, University of Rhode Island, 7 Greenhouse Road, Kingston, RI 02881, USA

${ }^{2}$ Nantong Maternal and Child Health Hospital, Nantong University, Nantong, China. ${ }^{3}$ Department of Epidemiology and Biostatistics, School of Public Health, Nantong University, 226006 Nantong, Jiangsu Province, China. ${ }^{4}$ These authors contributed equally: Sangmin You, Ai-Min Cui, Syed F. Hashmi

凶email: dengr@uri.edu
} 
reterm birth (PTB) is defined as babies born before 37 weeks of pregnancy (gestation) and is the leading cause of perinatal mortality worldwide ${ }^{1,2}$. Surviving preterm infants are also at risk for neurological, respiratory, and gastrointestinal complications; metabolic syndrome; and cardiovascular disease $^{3-5}$. The prevalence of PTB ranges from $5 \%$ to $18 \%$ globally $^{6}$. In the United States, one out of ten newborns is delivered preterm and the PTB rates exhibit an uptrend with unknown reasons ${ }^{7}$.

The underlying mechanisms by which PTB is induced are complex and multi-factorial ${ }^{8,9}$. Despite current research efforts, our understanding on the underlying mechanisms for PTB remains limited. As a consequence, current available interventions for preventing PTB show inconsistent benefits ${ }^{10,11}$. Bile acids are synthesized in the liver and their homeostasis is maintained through a tightly regulated enterohepatic circulation mainly through the complex farnesoid X receptor (FXR) signaling pathways ${ }^{12,13}$. Bile acids are recently recognized as signaling molecules to regulate a variety of cellular and metabolic activities $^{14-16}$. In the studies of pregnant women with intrahepatic cholestasis of pregnancy (ICP), it is recognized that elevated bile acids are associated with increased risk for $\mathrm{PTB}^{17-19}$. However, a direct etiological link between bile acids and PTB has not been established and it remains to be determined whether liver injury itself or elevated bile acids as a result of liver injury induce PTB.

In this study, a hospital-based cohort study with pregnant women is conducted. PTB rates and serum total bile acids (sTBAs) are determined and their correlations are evaluated. Using mouse models, PTB is successfully reproduced with liver injuries or bile acid challenge alone. Furthermore, restoring bile acid homeostasis by FXR activation markedly reduces PTB and dramatically improves newborn survival. The findings establish a direct etiological link between bile acids and PTB and open an avenue for developing mechanism-based therapies to prevent or delay PTB.

\section{Results}

Participant characteristics. Among the 36,755 pregnant women enrolled in the study, 36,612 subjects were ethnic Han Chinese women $(99.61 \%)$, while 143 subjects were other ethnic minority women $(0.39 \%)$. Twenty-two thousand five hundred and seventyone women $(61.41 \%)$ were primipara, while 14,184 women (38.59\%) were multipara. Among the multipara, 652 women had a history of PTB, while 23 women had a history of stillbirth. The patients diagnosed with ICP received ursodeoxycholic acid at a dose of $15 \mathrm{mg} \mathrm{kg}^{-1}$ day $^{-1}$ until delivery. The cervical length of the subjects with PTB was not evaluated. The average gestation age when PTB occurred was $34 \pm 2.3$ weeks. Most of the PTBs occurred at the gestation ages between 33 and 36 weeks $(n=$ $2496,86.8 \%)$ with 55 subjects (1.9\%) having PTB at gestation ages $<28$ weeks and 324 women $(11.3 \%)$ having PTB at gestation ages 28-32 weeks.

sTBA levels positively correlated with the rates of PTB. Among 36,755 pregnant women, 2875 subjects experienced PTB while 33,880 subjects had full-term birth (FTB). The sTBAs were significantly increased from $4.68 \pm 5.31 \mu \mathrm{M}$ in FTB to $5.69 \pm 12.45$ $\mu \mathrm{M}$ in PTB subjects $\left(p=4.2 \times 10^{-18}\right)$ (Fig. 1a). When sTBAs were elevated from $<10 \quad(n=34,227)$ to $10-39.9(n=2362)$, $40-99.9(n=146)$, and $\geq 100 \mu \mathrm{M}(n=20)$, the rates of PTB were significantly increased from $7.6 \%$ to $9.2 \%$ (relative risk (RR) 1.21 , 95\% confidence interval (CI) $1.05-1.39)(p=0.0055), 29.5 \%$ (RR 3.87 , 95\% CI 2.86-5.23) $\left(p=5.3 \times 10^{-24}\right)$, and 50\% (RR 6.57, $95 \%$ CI 3.53-12.22) $\left(p=9.6 \times 10^{-14}\right)$, respectively (Fig. $1 \mathrm{~b}$ and Supplementary Table 1). Correlation analysis confirmed that
sTBA concentrations directly correlated with the PTB rates $(r=$ $0.988 ; p=0.012$; Fig. $1 b)$.

PTB is clinically categorized into spontaneous (sPTB) and iatrogenic (medically indicated) PTB (iPTB). Among the 2875 PTB subjects, 1302 had sPTB (45.3\%) while 1573 had iPTB (54.7\%). Compared with FTB subjects, the sTBAs were significantly elevated in subjects with $\operatorname{sPTB}(p=0.0027)$ and iPTB subjects $\left(p=2.6 \times 10^{-22}\right.$; Supplementary Fig. 1a). The sPTB rates were significantly raised from $3.5 \%$ in subjects with $<10 \mu \mathrm{M}$ sTBAs to $10.3 \%$ in subjects with sTBAs $40-99.9 \mu \mathrm{M}$ (RR 2.9, 95\% CI $1.74-4.83 ; p=0.0001)$ and to $20 \%$ in subjects with sTBAs $\geq 100 \mu \mathrm{M}$ (RR 5.65, 95\% CI 2.12-15.07; $p=0.005$ ) (Supplementary Fig. $1 \mathrm{~b}$ and Supplementary Table 1). Consistently, sTBA levels positively correlated with the sPTB rates $(r=0.988, p=$ 0.007; Supplementary Fig. 1b). Similar to sPTB, the iPTB rates significantly increased from $4.2 \%$ to $6.2 \%$ (RR $1.51,95 \% \mathrm{CI}$ $\left.1.28-1.80 ; p=7.6 \times 10^{-8}\right), 19.2 \%$ (RR $4.71,95 \%$ CI $3.24-6.85$; $\left.p=5.7 \times 10^{-21}\right)$, and $30 \%$ (RR 7.37, 95\% CI 3.31-16.44; $p=$ $\left.4.7 \times 10^{-10}\right)$, respectively, when sTBA concentrations were elevated from $<10$ to $10-39.9,40-99.9$, and $\geq 100 \mu \mathrm{M}$ (Supplementary Fig. 1c and Supplementary Table 1). sTBA levels were also directly correlated with the iPTB rates $(r=0.978, p=0.002$; Supplementary Fig. 1c).

Consistent with sTBA, aspartate aminotransferase (AST) levels, which serve as the markers for liver injuries, were significantly increased from $16.36 \pm 14.70 \mathrm{UL}^{-1}$ in FTB subjects to $20.35 \pm$ $28.56 \mathrm{U} \mathrm{L} \mathrm{L}^{-1}$ in PTB subjects $\left(p<10^{-31}\right.$; Fig. 1c). When AST levels increased from $0-40(n=35,360)$ to $41-80(n=1046), 81-200$ $(n=284)$, and $>200 \mathrm{UL}^{-1} \quad(n=65)$, the PTB rates were significantly increased from $7.57 \%$ to $11.76 \%\left(p=1.3 \times 10^{-19}\right)$, $19.37 \%\left(p<10^{-31}\right)$, and $30.77 \%\left(p=1.8 \times 10^{-12}\right)$, respectively (Fig. 1d). AST levels directly correlated with the PTB rates $(r=$ $0.985, p=0.015$; Fig. 1d) and sTBA concentrations (Fig. 1e). Consistently, serum AST levels were significantly increased in subjects with $\operatorname{sPTB}\left(p=1.7 \times 10^{-5}\right)$ and $\operatorname{iPTB}\left(p<10^{-31}\right)$ and directly correlated with the rates of sPTB $(r=0.968, p=0.032)$ and iPTB $(r=0.964, p=0.036)$ (Supplementary Fig. 1d-f). In addition, a positive correlation was detected between serum AST and sTBA levels in subjects with $\operatorname{sPTB}(r=0.98, p=0.02$; Supplementary Fig. 1g). However, no significant correlation was detected between serum AST and TBA levels in subjects with iPTB ( $r=0.909, p=0.091$; Supplementary Fig. $1 \mathrm{~h})$.

Consistent with findings for AST, the PTB rates were significantly increased when other liver injury markers were elevated. As alanine aminotransferase (ALT) levels were elevated from $0-40$ to $41-80,81-200$, and $>200 \mathrm{UL}^{-1}$, the PTB rates were increased from $8.8 \%$ to $9.6 \%(p=0.25)$, $14.0 \%\left(p=5 \times 10^{-5}\right)$, and $27.7 \%\left(p=2.2 \times 10^{-17}\right)$, respectively (Supplementary Fig. 1i). When total bilirubin (TBiL) concentrations were elevated from $<17.1$ to $17.2-34.2 \mu \mathrm{M} \mathrm{L}^{-1}$, the PTB rates remains unchanged. However, when TBiL concentrations were further elevated to $>34.3 \mu \mathrm{M} \mathrm{L}^{-1}$, the PTB rates were markedly increased from $8.7 \%$ to $37.5 \% \quad\left(p=1.3 \times 10^{-12}\right.$; Supplementary Fig. 1j). Similarly, as serum gamma-glutamyl transpeptidase (GGT) levels elevated from $<50$ to $51-100$ and $>100 \mathrm{U} \mathrm{L}^{-1}$, the PTB rates were significantly increased from $8.8 \%$ to $23.3 \%\left(p=2.2 \times 10^{-20}\right)$ and $23.7 \%\left(p=3.4 \times 10^{-9}\right)$, respectively (Supplementary Fig. 1k).

Characteristics of pregnant women, sTBA levels, and PTB rates. Among the characteristics of the subjects, maternal age ${ }^{20-23}$, and pre-pregnancy body mass index (BMI) ${ }^{24-27}$ had significant impacts on PTB. We performed correlation analyses between sTBA concentrations and PTB rates in subjects with different maternal ages or pre-pregnancy BMIs. 

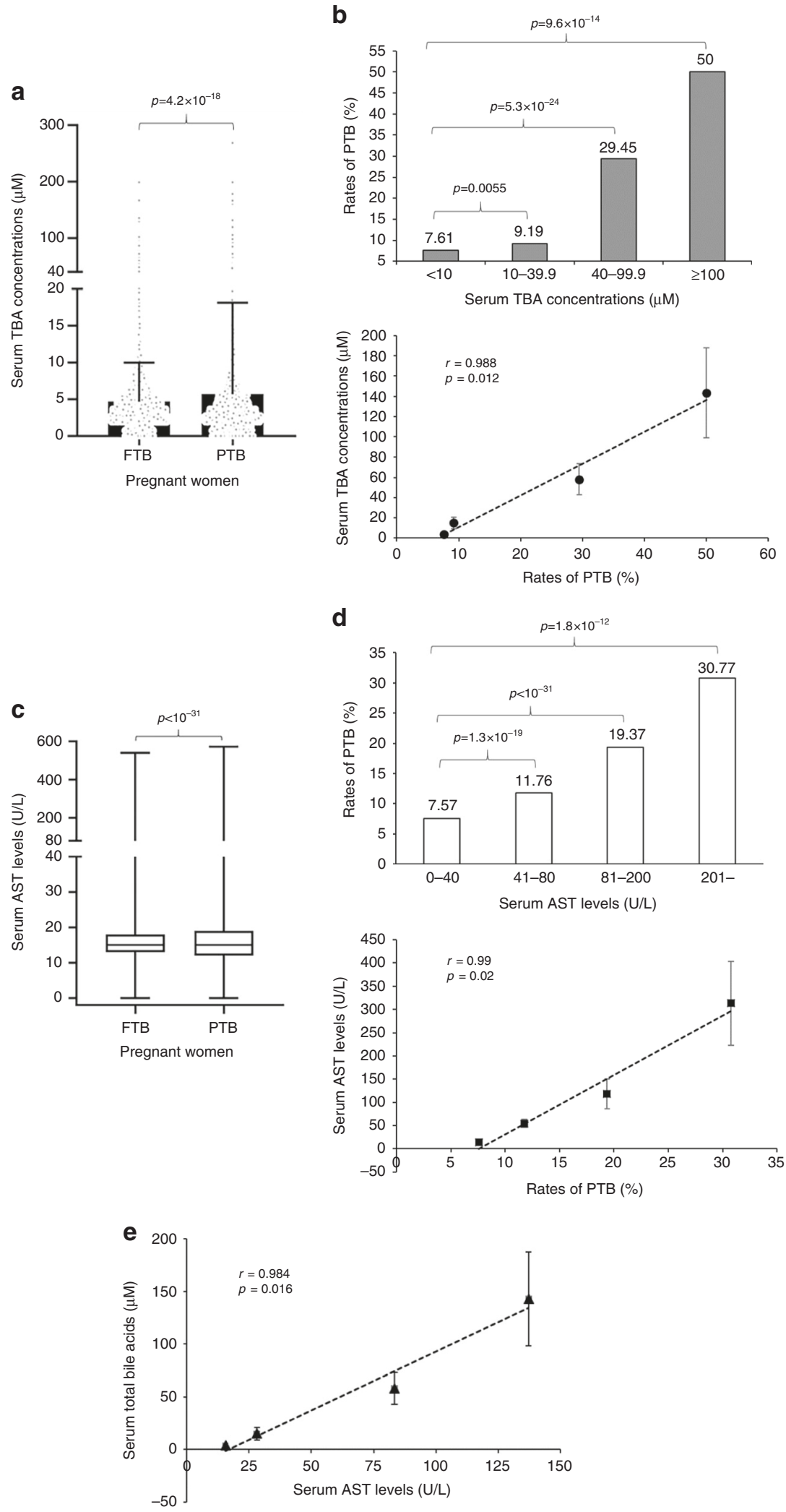

The subjects were divided into five age groups, 18-24, 25-29, $30-34,35-39$, and $40-50$ years. Subjects at ages $25-29$ years had the largest population $(n=19,289)$ and the lowest PTB rate (6.8\%) (Fig. 2a and Supplementary Table 2). As the maternal age advanced, the PTB rates increased from $6.8 \%$ to $8.6 \%(p=2.4 \times$ $\left.10^{-6}\right), 11.5 \%\left(p=2.9 \times 10^{-15}\right)$, and $20.1 \%\left(p=6.1 \times 10^{-21}\right)$ in the age groups $30-34(n=6122), 35-39(n=2025)$, and $40-50$ $(n=319)$, respectively. The youngest age group (age $18-24, n=$ $9000)$ also had a significantly increased PTB rate $(8.1 \%)(p=$ $\left.1.3 \times 10^{-4}\right)$. sTBAs showed an uptrend as maternal age advanced and directly correlated with the PTB rates among the five age groups ( $r=0.981, p=0.003$; Fig. $2 b$ ).

Based on the BMIs, the subjects were categorized into four groups. Among the 36,755 pregnant women, $78.91 \%$ subjects had 
Fig. 1 Elevated sTBAs in pregnant women with PTB. a sTBA levels in pregnant women with FTB $(n=33,880)$ and PTB $(n=2875)$. Bar chart shows the mean \pm SD with small dots representing the data point distribution. b As sTBA levels elevated from $<10(n=34,227)$ to $10-39.9(n=2362), 40-99.9(n=$ $146)$ and $\geq 100 \mu \mathrm{M}(n=20)$, the PTB rates gradually increased. The PTB rates directly correlated with the sTBA concentrations. Data points in the lower panel are presented as mean \pm SD. c Serum AST levels in pregnant women with FTB $(n=33,880)$ and PTB $(n=2875)$. The box and whisker plots show the maximum, minimum data points, median value, and 75th and 25th quartile. d Various AST levels of 0-40 $(n=35,360), 41-80(n=1046), 81-200(n=$ 284), and $>200 \cup L^{-1}(n=65)$ with corresponding PTB rates. The PTB rates directly correlated with AST levels. Data points in the lower panel are presented as mean \pm SD. e sTBA concentrations of $<10(n=34,227), 10-39.9(n=2362), 40-99.9(n=146)$, and $\geq 100 \mu \mathrm{M}(n=20)$ directly correlated with the AST levels. Data points are presented as mean \pm SD. Student's $t$ test for pairwise comparison (two-sided) and one-way ANOVA, followed by Tukey post hoc test were applied for statistical analysis. Pearson correlation analysis was applied to determine the correlation coefficient and associated $p$ values. Source data are provided as a Source Data file.

healthy body weight (BMI 18.5-24.9, $n=29,004)$ with the lowest PTB rate of $7.1 \%$ (Fig. 2c). When pregnant women become overweight (BMI 25-29.9, $n=4593$ ) and obese (BMI $\geq 30, n=$ $185)$, the PTB rates were significantly increased to $11.9 \%(p=$ $\left.8.9 \times 10^{-31}\right)$ and $14.6 \%\left(p=7.9 \times 10^{-5}\right)$, respectively. On the other hand, underweight subjects (BMI $<18.5, n=2973)$ also had a significantly increased PTB rate of $8.2 \%(p=0.03)$. More importantly, sTBAs exhibited an increased trend as BMI increased and directly correlated with the PTB rates among the four BMI groups $(r=0.949 ; p=0.05)$.

Similar to sTBA, AST levels gradually increased as maternal age advanced or BMI increased and positively correlated with the PTB rates $(r=0.988, p=0.002$ or $r=0.966, p=0.034$, respectively; Supplementary Fig. 2a, b). As expected, the AST and sTBA levels were directly correlated among the five age groups $(r=$ $0.983, p=0.003)$ and four BMI groups $(r=0.976, p=0.024)$ (Supplementary Fig. 2c, d).

Liver disorders of pregnant women, sTBA levels, and PTB rates. Among the 36,755 subjects, 1805 were diagnosed having non-alcoholic fatty liver disease (NAFLD), a rate of $4.91 \%$, while 505 were diagnosed having ICP, a rate of $1.37 \%$. Compared with the rate of $7.82 \% \mathrm{PTB}$ in all the subjects, pregnant women with NAFLD and ICP had significantly increased PTB rates, $13.96 \%(p$ $\left.=3.1 \times 10^{-19}\right)$ and $23.17 \%\left(p<10^{-31}\right)$, respectively (Fig. 3a). Such increases in PTB rates correlated with elevated sTBA concentrations in those subjects. The sTBA levels were significantly elevated from $4.8 \pm 5.3 \mu \mathrm{M}$ in all subjects to $7.3 \pm 13.3 \mu \mathrm{M}$ in NAFLD $\left(p<10^{-31}\right)$ and $31.7 \pm 24.4 \mu \mathrm{M}$ in ICP subjects $\left(p<10^{-31}\right)$ (Fig. 3a). Among the subjects with NAFLD, the sTBA levels were significantly higher in subjects with PTB $(11.83 \pm 22.01 \mu \mathrm{M}$, $n=217)$ than those with FTB $(6.53 \pm 10.28 \mu \mathrm{M}, n=1337)$ $\left(p=1.1 \times 10^{-9}\right.$; Fig. 3b). Similarly, among the pregnant women with ICP, significantly higher sTBA levels were detected in PTB subjects $(42.92 \pm 42.44 \mu \mathrm{M}, n=117)$ when compared to FTB subjects $(28.33 \pm 24.37 \mu \mathrm{M}, n=388)\left(p=3.6 \times 10^{-6}\right.$; Fig. $\left.3 \mathrm{~b}\right)$.

Similarly, the rates of both sPTB and iPTB were significantly increased from $3.54 \%$ and $4.28 \%$ in all subjects to $5.47 \%$ ( $p=$ $\left.6.18 \times 10^{-6}\right)$ and $8.49 \%\left(p=3.0 \times 10^{-16}\right)$ in NAFLD subjects and $6.14 \%(p=0.0018)$ and $17.03 \%\left(p<10^{-31}\right)$ in ICP subjects, respectively (Supplementary Fig. $3 \mathrm{a}, \mathrm{b}$ and Supplementary Table 3). Correlatively, sTBAs were increased from $4.8 \pm 5.3 \mu \mathrm{M}$ in all subjects to $11.6 \pm 24.1\left(p=1.3 \times 10^{-6}\right)$ and $12.0 \pm 20.7 \mu \mathrm{M}$ $\left(p=3.0 \times 10^{-16}\right)$ in subjects with sPTB and iPTB among the NAFLD patients (Supplementary Fig. 3a, b). Similarly, sTBAs were markedly elevated to $46.5 \pm 44.4\left(p<10^{-31}\right)$ and $41.6 \pm 41.9$ $\mu \mathrm{M}\left(p<10^{-31}\right)$ in subjects with sPTB and iPTB, respectively, among the ICP patients (Supplementary Fig. 3a, b). Among the NAFLD subjects, sTBAs were significantly higher in subjects with sPTB $\left(11.57 \pm 24.06 \mu \mathrm{M}, n=85 ; p=10^{-4}\right)$ and $\mathrm{iPTB}(11.99 \pm$ $\left.20.68 \mu \mathrm{M}, n=132 ; p=2.8 \times 10^{-7}\right)$ than in the FTB subjects $(6.53$ $\pm 10.28 \mu \mathrm{M}, n=1337$ ) (Supplementary Fig. 3c). Similarly, among the subjects with ICP, sTBAs were significantly elevated from
$28.33 \pm 24.37 \mu \mathrm{M}$ in FTB subjects $(n=388)$ to $46.54 \pm 44.38 \mu \mathrm{M}$ in subjects with $\mathrm{sPTB}\left(n=31 ; p=2.0 \times 10^{-4}\right)$ and $41.62 \pm 41.92$ $\mu \mathrm{M}$ subjects with iPTB $\left(n=86 ; p=10^{-4}\right)$ (Supplementary Fig. 3c).

Consistent with liver injuries and increased sTBA levels in patients with NAFLD and ICP, serum AST levels were significantly elevated from $16.67 \pm 14.90 \mathrm{U} \mathrm{L}^{-1}$ in all the subjects to $28.72 \pm 35.45 \mathrm{U} \mathrm{L}^{-1}$ in NAFLD $\left(p<10^{-31}\right)$ and $72.40 \pm 85.31$ $\mathrm{U} \mathrm{L}^{-1}$ in ICP subjects $\left(p<10^{-31}\right)$. Elevated AST levels correlated with increased rates of PTB (Fig. 3c). Among the subjects with NAFLD, AST levels were significantly increased in subjects with PTB $\left(44.23 \pm 56.97 \mathrm{U} \mathrm{L}^{-1}\right)$ when compared to those with FTB $\left(26.20 \pm 34.51 \mathrm{U} \mathrm{L}^{-1}\right)\left(p=1.9 \times 10^{-10}\right.$; Fig. 3d). Similarly, higher AST levels were detected in PTB subjects $\left(83.16 \pm 91.38 \mathrm{U} \mathrm{L}^{-1}\right)$ than those with FTB $\left(69.15 \pm 84.22 \mathrm{UL}^{-1}\right)$ among ICP patients without reaching a statistical significance $(p=0.12$; Fig. $3 d)$. Consistently, among the NAFLD subjects, AST levels were significantly increased from $26.20 \pm 34.51 \mathrm{U} \mathrm{L}^{-1}$ in FTB subjects to $39.72 \pm 53.44 \mathrm{UL}^{-1}$ in $\operatorname{sPTB}\left(p=7.8 \times 10^{-4}\right)$ and $47.13 \pm$ $59.14 \mathrm{U} \mathrm{L}^{-1}$ in iPTB subjects $\left(p=10^{-9}\right)$ (Supplementary Fig. $3 \mathrm{~d}$ ). Similarly, among ICP subjects, serum AST levels were elevated in subjects with sPTB $\left(84.58 \pm 90.34 \mathrm{UL}^{-1}\right)$ and $\mathrm{iPTB}(82.65 \pm$ 92.27 $\left.\mathrm{U} \mathrm{L}^{-1}\right)$ when compared to the FTB subjects $(69.15 \pm 84.22)$ without reaching a statistical significance (Supplementary Fig. 3d).

Estrogen induced cholestasis and PTB in pregnant mice. Previous investigations reported that elevated reproductive hormones including estrogens during the late stage of pregnancy contributed to the pathogenesis of $\mathrm{ICP}^{23-25}$. Indeed, estrogeninduced cholestasis as animal models for ICP has been well established in rodents ${ }^{26,27}$. However, no studies have been carried out to investigate the effects of estrogen-induced cholestasis on the outcome of pregnancy in rodent models. In this study, we carried out experiments to determine whether estrogen-induced cholestasis and bile acid dysregulation can reproduce the PTB observed in ICP patients. As shown in Fig. 4a, treatment of pregnant mice with synthetic estrogen 17 a-ethynylestradiol (EE2) starting at gestation day 16.3 significantly induced PTB. The mean gestation days were significantly decreased from $19.9 \pm$ 0.44 days in control mice $(n=9)$ to $18.76 \pm 1.15$ days in EE2treated mice $(n=12)(p=0.013)$. Among the treated mice, $58.3 \%$ gave PTB (earlier than 19.1 days) and a large portion of newborns were dead with a huge variation from mouse to mouse $(p=$ 0.0068 ). The rates of live birth ranged from $0 \%$ to $91.7 \%$ with an average of $34.44 \pm 29.78 \%$, which is significantly lower than the control $(p=0.0031$; Fig. $4 \mathrm{a})$. sTBAs were markedly elevated from $7.49 \pm 2.01 \mu \mathrm{M}$ in control to $31.91 \pm 3.12 \mu \mathrm{M}$ in treated mice $\left(p=3.5 \times 10^{-14}\right)$ and negatively correlated with gestation days $(r=-0.647, p=0.043)$ and the rates of live birth $(r=-0.601$, $p=0.03$ ) (Fig. 4b). As expected, AST levels were significantly elevated from $17.3 \pm 8.5 \mathrm{U} \mathrm{L}^{-1}$ in control to $87.3 \pm 19.2 \mathrm{U} \mathrm{L}^{-1}$ in treated mice $\left(p=5.9 \times 10^{-9}\right)$. The AST levels negatively 

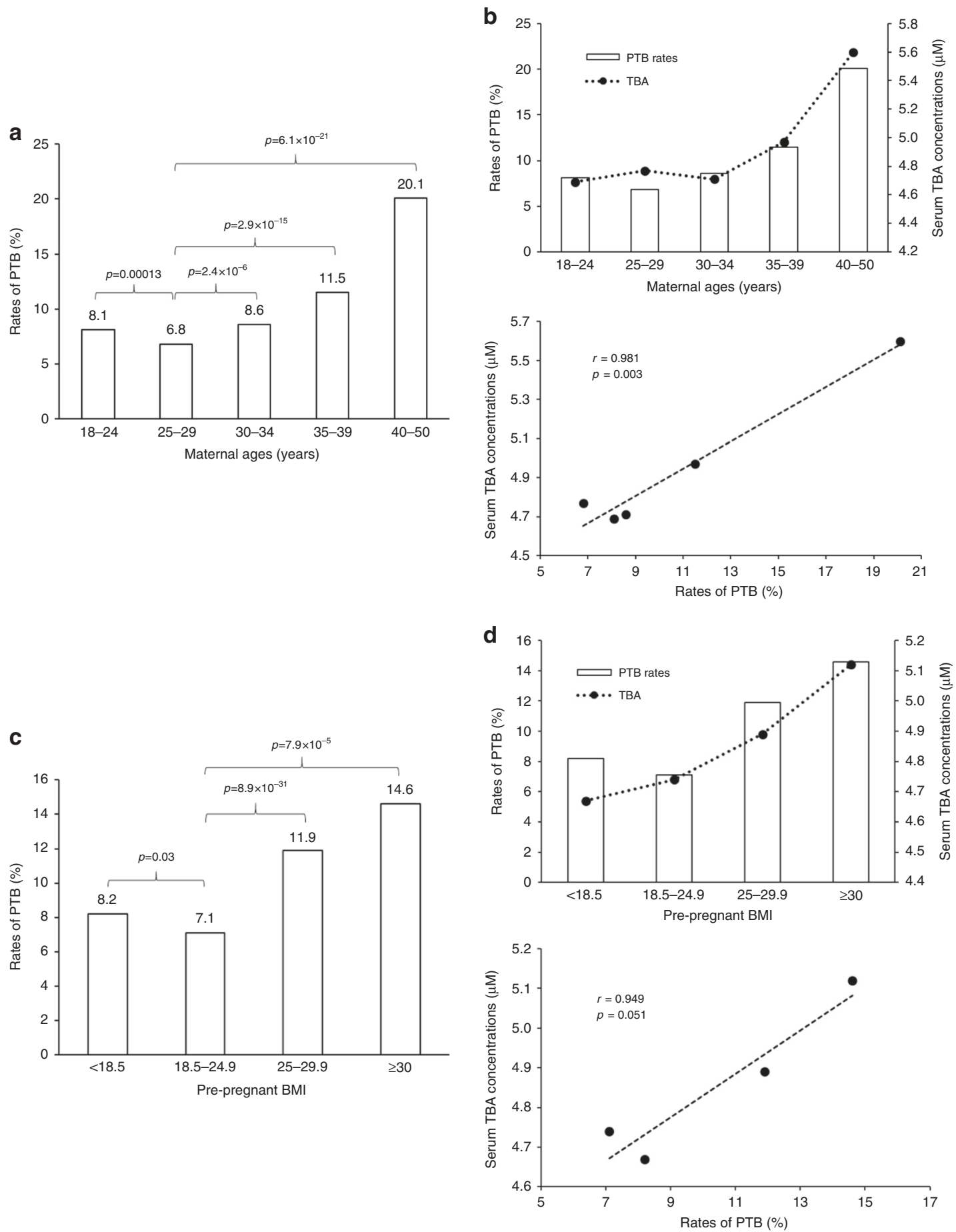

Fig. 2 Advanced maternal age and high pre-pregnancy BMI increased the risk for PTB with elevated sTBA. a The PTB rates of five groups of pregnant women at age 18-24 $(n=9,000), 25-29(n=19,289), 30-34(n=6122), 35-39(n=2025)$, and 40-50 $(n=319)$ years. b Serum TBA exhibited an uptrend as maternal age advanced and directly correlated with the PTB rates among the five age groups with the same sample sizes described in a. $\mathbf{c}$ The PTB rates of four groups of pregnant women with various BMIs, $<18.5(n=2973), 18.5-24.9(n=29,004), 25-29.9(n=4593)$. and $\geq 30(n=185)$.

d Serum TBA concentrations were elevated in overweight/obese subjects and positively correlated with the PTB rates among the four BMI groups with the same sample sizes described in c. One-way ANOVA, followed by Tukey post hoc test, and Pearson correlation test were applied for statistical analysis. Source data are provided as a Source Data file.

correlated with the rates of live birth $(r=-0.678, p=0.015)$ but not with gestation days $(r=0.143, p=0.657)$ and the sTBA concentrations $(r=0.135, p=0.675)$ (Supplementary Fig. 4a). In addition, no significant correlations were detected between the rates of live birth and the gestation days $(r=-0.177, p=0.583$; Supplementary Fig. 4b).
Carbon tetrachloride $\left(\mathrm{CCl}_{4}\right)$ induced liver injury and PTB in pregnant mice. Currently, no animal models have been established for studying PTB with liver injuries. In this study, we used $\mathrm{CCl}_{4}$ as a challenging agent to induce liver injury and bile acid dysregulation and investigate the effects on PTB in pregnant mice. $\mathrm{CCl}_{4}$ is a hepatotoxic agent that is commonly used to 

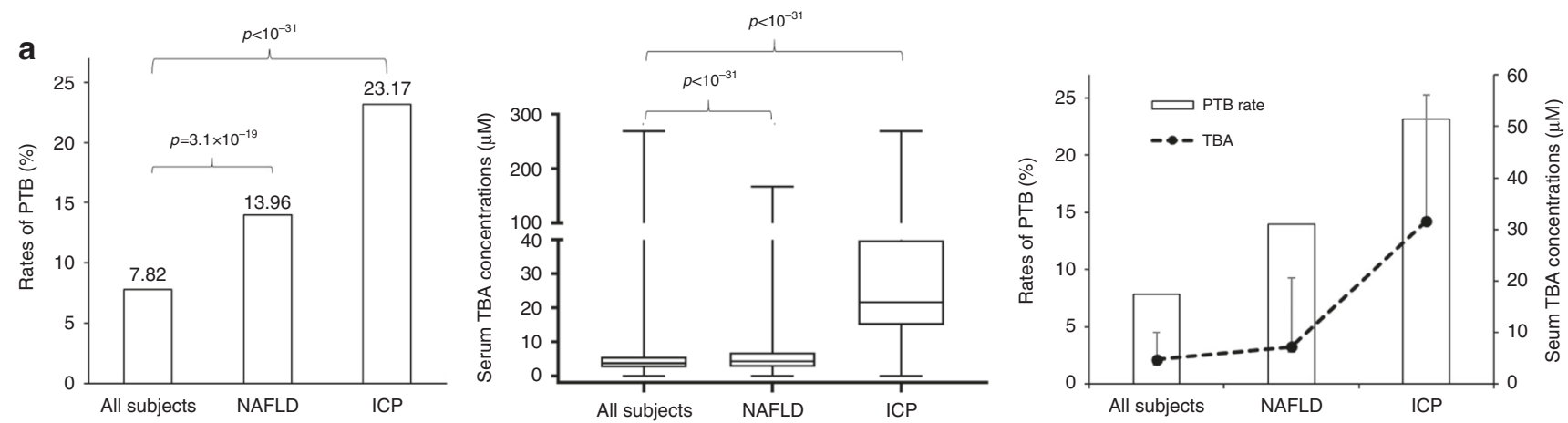

b
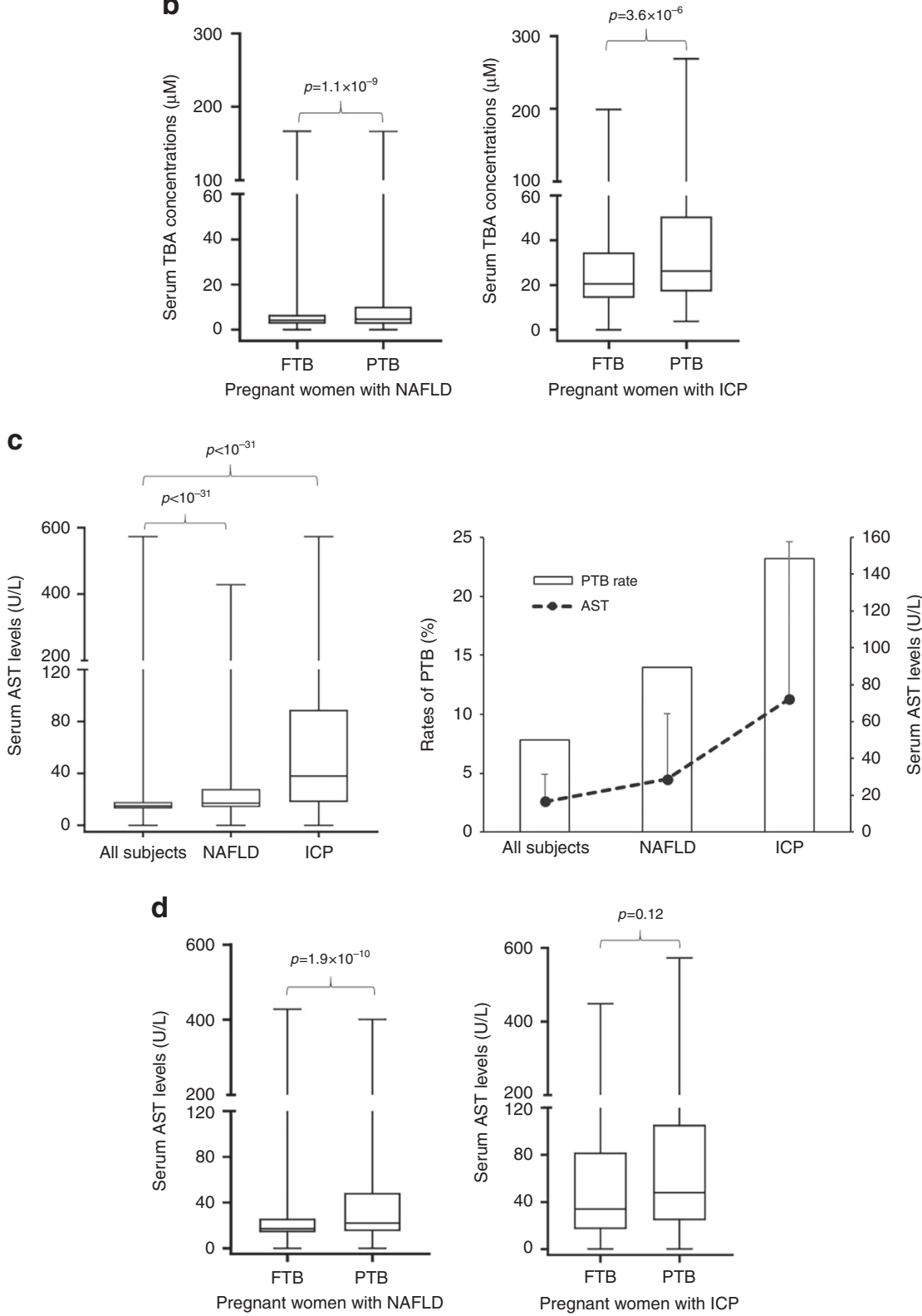

induce various hepatic injuries, including NAFLD, fibrosis, and non-alcoholic steatohepatitis.

As shown in Fig. 5a, $\mathrm{CCl}_{4}$ dose-dependently induced PTB. The mean gestation days were significantly shortened from $19.8 \pm$ 0.5 days in control $(n=9)$ to $18.9 \pm 1.1(p=0.036), 18.1 \pm 0.6(p=$ $\left.7.3 \times 10^{-7}\right)$, and $17.6 \pm 0.5$ days $\left(p=2.7 \times 10^{-6}\right)$ in mice treated with $1(n=5), 1.5(n=9)$, and $2 \mathrm{ml} \mathrm{kg}^{-1} \mathrm{CCl}_{4}(n=5)$, respectively.

Dosing of $1 \mathrm{ml} \mathrm{kg}^{-1} \mathrm{CCl}_{4}$ resulted in $60 \% \mathrm{PTB}$ while $100 \%$ mice gave PTB with the doses of 1.5 and $2 \mathrm{ml} \mathrm{kg}^{-1} \mathrm{CCl}_{4}$. The minimal dose $\left(1.5 \mathrm{ml} \mathrm{kg}^{-1}\right)$ that induced $100 \%$ PTB was used for subsequent studies, and the mice in this group were further characterized. The rates of live birth were markedly decreased to $26.63 \pm 27.82 \%$ in mice treated with $1.5 \mathrm{ml} \mathrm{kg}^{-1} \mathrm{CCl}_{4}$ ( $p=0.0015$; Fig. 5a). sTBAs were significantly elevated from $7.49 \pm 2.01 \mu \mathrm{M}$ in control to 
Fig. 3 Pregnant women with NAFLD and ICP had increased risk for PTB with elevated sTBAs. a The PTB rates, sTBA levels, and their correlations in al subjects $(n=36,755)$ and the subjects with NAFLD $(n=1554)$ or ICP $(n=505)$. The box and whisker plots of the middle panel show the maximum, minimum data points, median value, and 75th and 25th quartile. Data points for serum TBA are presented as mean \pm SD in the right panel. $\mathbf{b}$ The sTBA levels in the subjects with FTB ( $n=1337$ for NAFLD, $n=388$ for ICP) and PTB ( $n=217$ for NAFLD, $n=117$ for ICP) among the pregnant women with NAFLD or ICP. The box and whisker plots show the maximum, minimum data points, median value, and 75th and 25th quartile. c Serum AST levels in all subjects $(n=36,755)$ and subjects with NAFLD $(n=1554)$ or ICP $(n=505)$. The AST levels correlated with the rates of PTB. The box and whisker plots in the left panel show the maximum, minimum data points, median value, and 75th and 25th quartile. The data points for AST levels are presented as mean \pm SD in the right panel. d The AST levels in subjects with FTB ( $n=1337$ for NAFLD and $n=388$ for ICP) and PTB $(n=217$ for NAFLD and $n=117$ for ICP) among the pregnant women with NAFLD or ICP. The box and whisker plots show the maximum, minimum data points, median value, and 75th and 25th quartile. Student's $t$ test for pairwise comparison or one-way ANOVA, followed by Tukey post hoc test for multiple group comparison were applied for statistical analysis. Source data are provided as a Source Data file.
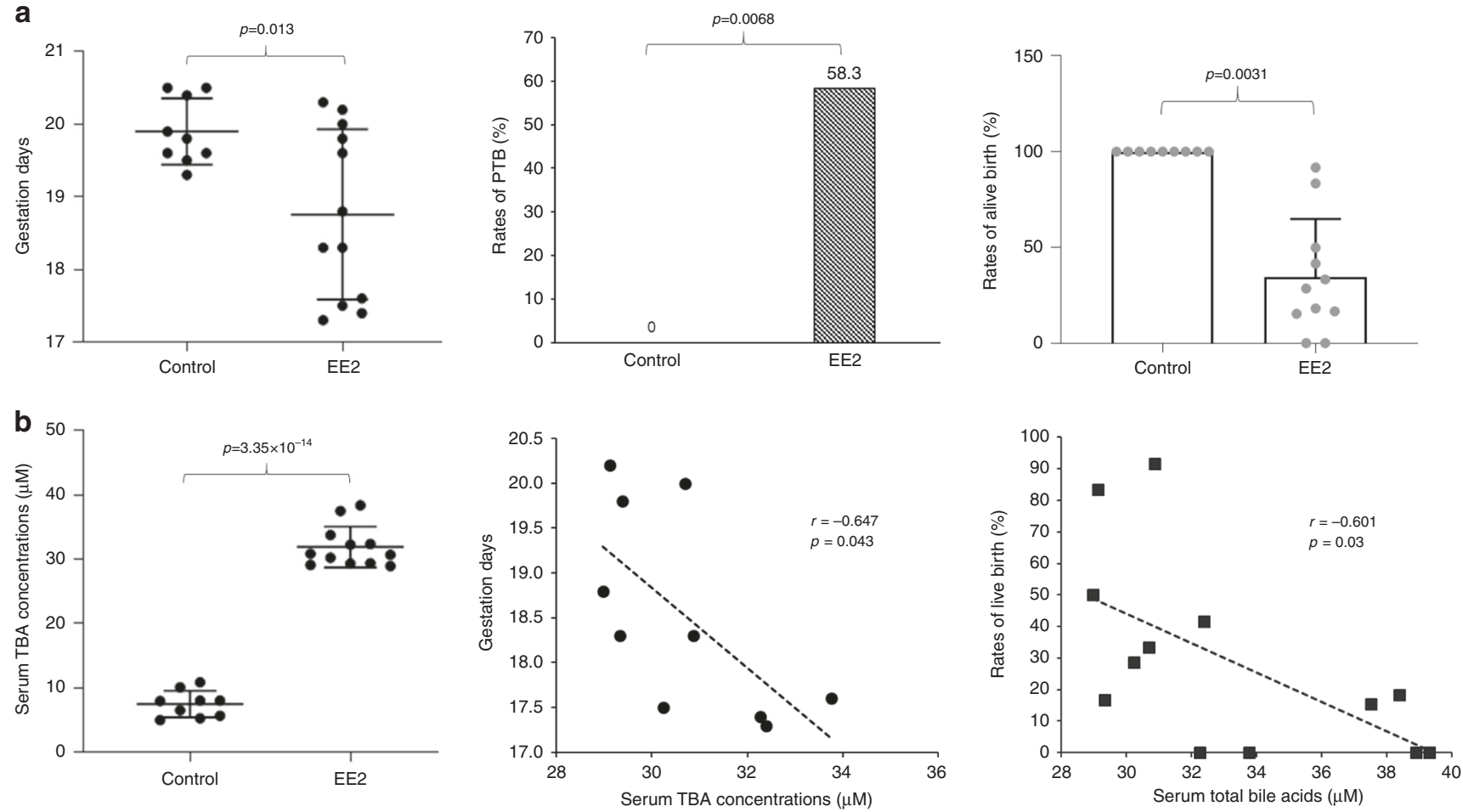

Fig. 4 EE2-induced liver injuries, bile acid dysregulation, and PTB in pregnant mice. a The gestation days, PTB rates, and rates of live birth in controls $(n=9)$ and EE2-treated pregnant mice $(n=12)$. The data are presented as mean \pm SD in the right and left panels. $\mathbf{b}$ The sTBA levels in controls $(n=9)$ and EE2-treated mice $(n=12)$ and a negative correlation between STBA concentrations and the gestation days or the rates of live birth. The means and standard errors of the group values were indicated by the long and short lines, respectively. Student's $t$ test for pairwise comparison (two-sided), Chisquared test for proportion comparisons, and Pearson correlation analysis were applied for statistical analysis. Source data are provided as a Source Data file.

$27.78 \pm 10.02 \mu \mathrm{M}$ in treated mice $\left(p=1.7 \times 10^{-5}\right)$ and negatively correlated with the gestation days $(r=-0.867, p=0.002)$ as well as the rates of live birth $(r=-0.848, p=0.004)$ (Fig. 5b).

As expected, AST concentrations were markedly elevated from $17.3 \pm 8.5 \mathrm{U} \mathrm{L}^{-1}$ in control to $122.1 \pm 22.8 \mathrm{U} \mathrm{L}^{-1}$ in $\mathrm{CCl}_{4}$-treated mice $\left(p=1.1 \times 10^{-9}\right)$ and positively correlated with sTBA levels $(r=0.765, p=0.016)$. However, no significant correlations were detected between AST levels and the gestation days $(r=-0.545$, $p=0.129)$ nor with the rates of live birth $(r=-0.49, p=0.181$; Supplementary Fig. 5a). It was noted that most of the dead newborns were from the mice having extremely early PTB around gestation day 17.5. Consistently, the rates of live birth positively correlated with the gestation days $(r=0.967, p<0.001$; Supplementary Fig. 5b).

Bile acid cholic acid (CA) induced PTB in pregnant mice. The data from pregnant women and mice treated with $\mathrm{EE} 2$ or $\mathrm{CCl}_{4}$ all demonstrated that sTBA levels positively correlated with the PTB rates. We hypothesized that elevated serum bile acids directly induced PTB. To test the hypothesis, pregnant mice were treated with a diet containing $0.5 \%$ or $1 \%$ CA starting at gestation day 15.3. As shown in Fig. 6a, CA dose-dependently induced PTB. The average gestation days were decreased from $19.9 \pm 0.4$ in the control group $(n=9)$ to $19.4 \pm 0.8$ with a PTB rate of $50 \%$ in mice fed with $0.5 \%$ CA diet $(n=8 ; p=0.14)$ and $17.9 \pm 0.3$ with a PTB rate of $100 \%$ in mice fed with $1 \%$ CA diet $\left(n=6 ; p=5.1 \times 10^{-7}\right)$. All the newborns were alive in mice fed with $0.5 \%$ CA diet while on average $85.0 \pm 10.6 \%$ of newborns were alive in the group fed with $1 \%$ CA.

As expected, sTBA levels were significantly elevated in the two treatment groups. The average sTBAs were increased from $7.49 \pm$ $2.01 \mu \mathrm{M}$ in control mice to $17.4 \pm 3.3 \mu \mathrm{M}$ in mice fed with $0.5 \%$ CA diet $\left(p=2.7 \times 10^{-6}\right)$ and $24.9 \pm 7.2 \mu \mathrm{M}$ in mice fed with $1 \%$ CA diet $\left(p=1.5 \times 10^{-5}\right)$ (Fig. $\left.6 \mathrm{~b}\right)$. Correlation analysis revealed that sTBAs negatively correlated with the gestation days 

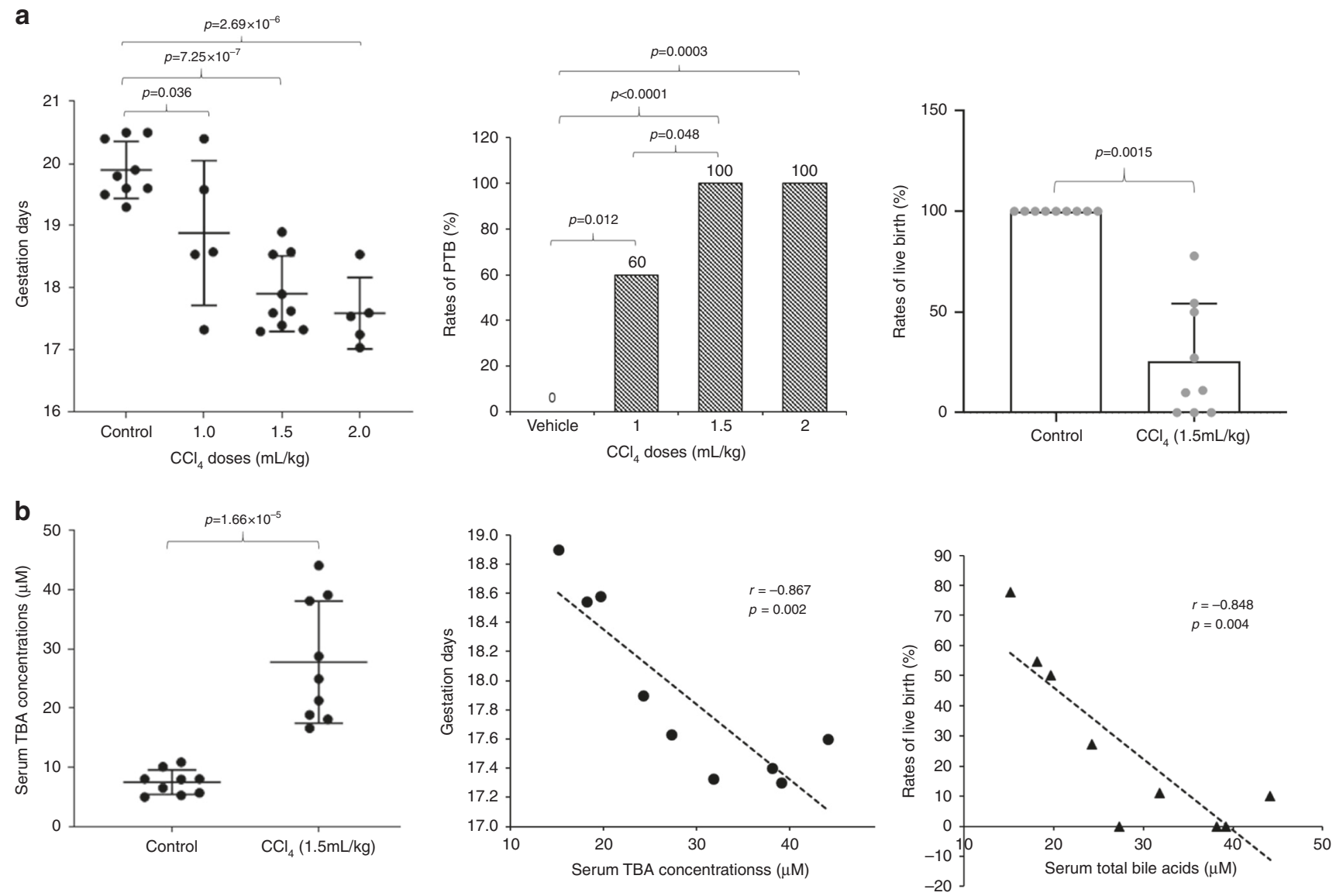

Fig. $5 \mathbf{C C l}_{\mathbf{4}}$-induced liver injuries, bile acid dysregulation, and PTB in pregnant mice. a The gestation days, PTB rates and rates of live birth in controls $(n=9)$ and pregnant mice treated with $1.0(n=5), 1.5(n=9)$, or $2 \mathrm{ml} \mathrm{kg}^{-1} \mathrm{CCl}_{4}(n=5)$. b The sTBA levels in controls $(n=9)$ and mice treated with $1.5 \mathrm{~kg} \mathrm{ml}^{-1} \mathrm{CCl}_{4}(n=9)$ and a negative correlation between STBA concentrations and gestation days or the rates of live birth. The means and standard errors of the group values were indicated by the long and short lines, respectively. Student's $t$ test for pairwise comparison, one-way ANOVA followed by Tukey post hoc test for multiple group comparison, Chi-squared test for proportion comparisons and Pearson correlation test were applied for statistical analysis. Source data are provided as a Source Data file.

$(r=-0.801, p=0.001)$ and the rates of live birth $(r=-0.803, p$ $=0.001$ ) (Fig. 6b).

Consistent with CA being a mild bile acid in inducing hepatotoxicity, AST levels were only slightly increased from $17.3 \pm 8.5 \mathrm{U} \mathrm{L}^{-1}$ in control to $22.8 \pm 4.6(p=0.14)$ and $32.1 \pm 7.9$ $\mathrm{UL}^{-1}(p=0.006)$ in CA-treated mice (Fig. $\left.6 \mathrm{c}\right)$. It should be noted that the AST levels among the three groups are all within the normal physiological range for mice (from 10 to $45 \mathrm{U} \mathrm{L}^{-1}$ ), indicating that minimal liver injury was induced by CA treatment. Correlation analysis showed that AST levels negatively correlated with gestation days $(r=-0.835, p<0.001)$ and the rates of live birth $(r=-0.892, p<0.001$; Supplementary Fig. 6a) while positively correlated with sTBAs $(r=0.957, p<0.001$; Supplementary Fig. 6a). A direct correlation between the gestation days and rates of live birth was also detected ( $r=$ $0.774, p=0.002$; Supplementary Fig. $6 \mathrm{~b}$ ).

Elevated bile acids, not liver injury, induced PTB. As presented above, $0.5 \%$ CA in diet induced 50\% PTB with minimal liver injury as AST levels did not differ from the controls. The data indicated that it was the elevated bile acids, not liver injury, that induced PTB. To further confirm such notion, we carried out additional studies to elevate bile acid levels without inducing liver injury. First, we treated the non-pregnant mice with a reduced dose of CA $(0.3 \%)$ or FXR antagonist DY268 to determine the effects of the treatments on sTBA and AST levels. It was reasoned that FXR antagonism would increase endogenous bile acid levels through increasing bile acid synthesis and reducing bile acid disposition. As shown in Supplementary Fig. 7a, sTBA levels were significantly elevated in mice treated with $0.3 \%$ CA in diet $\left(n=7 ; p=7.2 \times 10^{-5}\right)$ or DY268 $\left(n=7 ; p=9.4 \times 10^{-5}\right)$ when compared to the controls $(n=9)$. However, the AST levels were comparable to the control group $(p=0.97$ or $p=0.92$; Supplementary Fig. 7b), indicating that no liver injury was induced by either of the treatments. The bile acid levels in mice treated with $0.3 \%$ CA in diet or DY268 were elevated to $14.1 \pm$ 2.6 or $13.7 \pm 2.4 \mu \mathrm{M}$, which may not be high enough to effectively induce PTB based on the findings with the treatment of $0.5 \% \mathrm{CA}$ in diet (Fig. 6a, b). Therefore, we treated pregnant mice with a combination of $0.3 \% \mathrm{CA}$ and DY268. As shown in Fig. 7a, the average gestation days were significantly decreased from $19.9 \pm$ 0.4 in the control group $(n=9)$ to $18.3 \pm 0.3$ in the treatment groups $(n=8)\left(p=2.1 \times 10^{-5}\right)$. A rate of $87.5 \%$ PTB (7 out of 8 mice) was reached in the pregnant mice treated with $0.3 \% \mathrm{CA}$ and DY268 $\left(p=4 \times 10^{-4}\right)$. As expected, sTBA levels were markedly elevated to $22.3 \pm 2.8 \mu \mathrm{M}$ in the treatment group ( $p=$ $3.1 \times 10^{-9}$; Fig. $7 \mathrm{~b}$ ), which is significantly higher than the sTBA levels in mice treated with $0.3 \%$ CA or DY268 alone (Supplementary Fig. 7a). On the other hand, AST levels were comparable to the control group ( $p=0.33$; Fig. $7 c)$. Histopathology examination confirmed no liver injury (Fig. $7 d$ ). Consistent with the findings from pregnant mice treated with $0.5 \%$ (Fig. $6 \mathrm{a}$ ), all the 
a
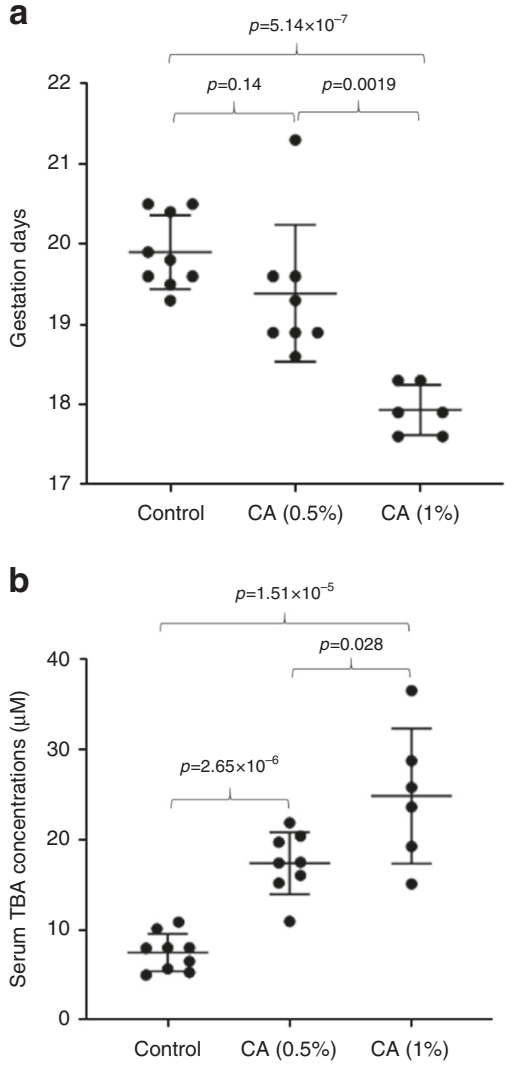
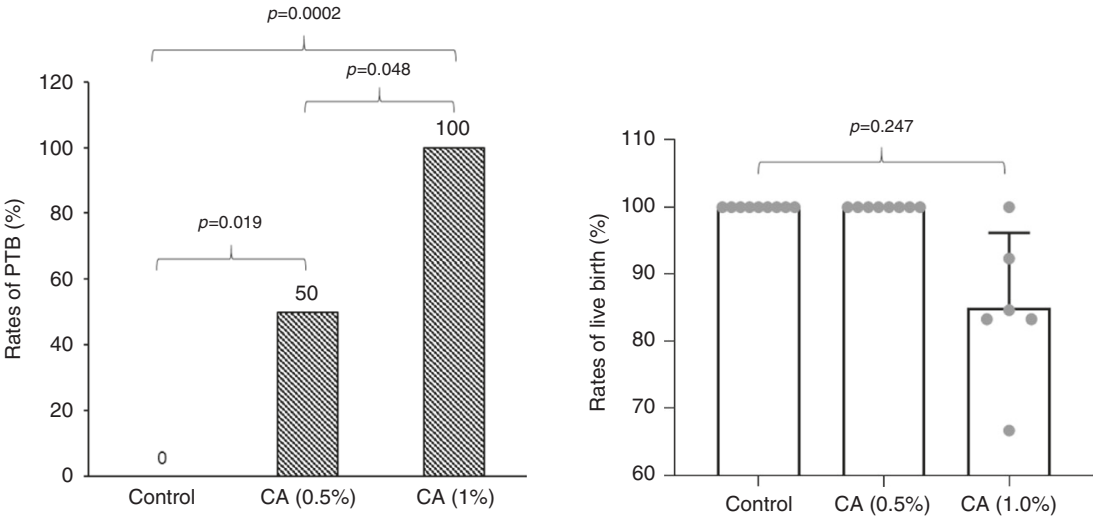
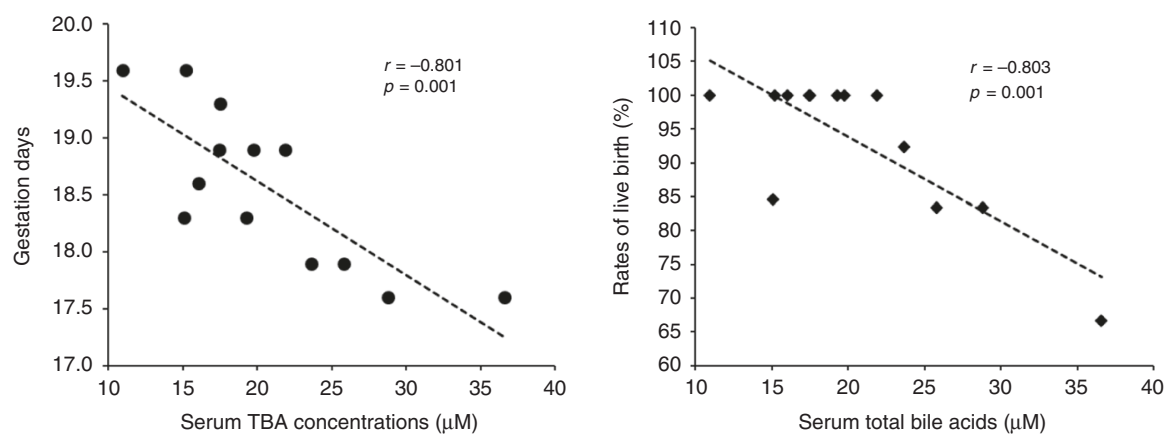

C
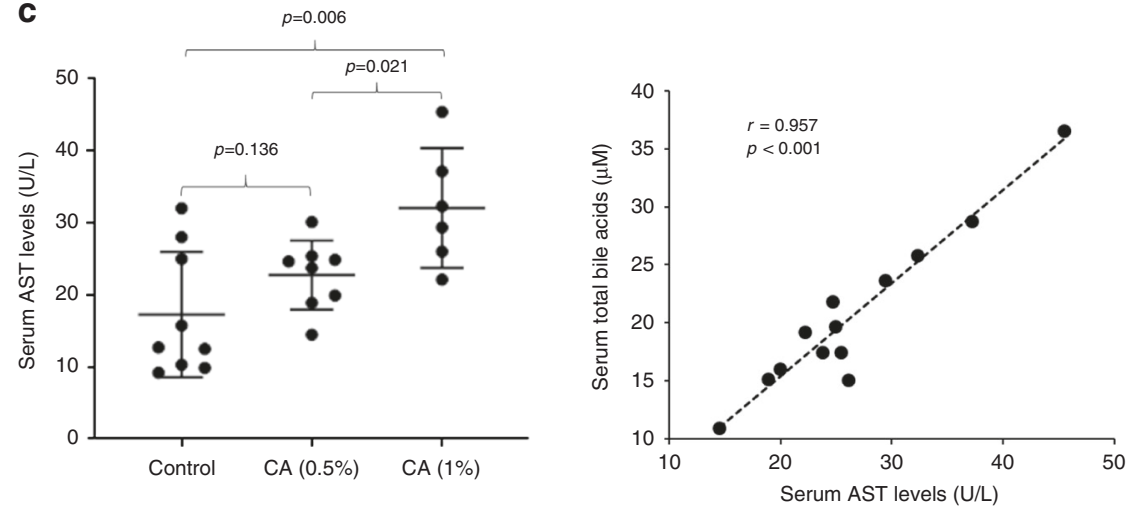

Fig. 6 Bile acid CA effectively induced PTB in pregnant mice. a The gestation days, PTB rates, and the rates of live birth in control ( $n=9)$ and mice treated with a diet containing $0.5 \%(\mathrm{w} / \mathrm{w})(n=8)$, or $1 \%$ CA $(n=6)$. b The sTBA levels in controls $(n=9)$ and mice treated with CA diet $(n=13)$ and a negative correlation between the sTBA levels and the gestation days or the rates of live birth. c Serum AST levels in controls $(n=9)$ and CA-treated mice $(n=13)$ and a direct correlation between STBA and AST levels in CA-treated mice. The means and standard errors of the group values were indicated by the long and short lines, respectively. One-way ANOVA, followed by Tukey post hoc test and Chi-squared test for proportion comparisons were applied for statistical analysis. Pearson correlation analysis was applied to determine the correlation coefficient and associated $p$ values. Source data are provided as a Source Data file.

newborns were alive. The data thus firmly established that it was the elevated bile acids, not liver injury itself, that induced PTB.

Prevention of PTB by restoring bile acid homeostasis. FXR is the master regulator of bile acid homeostasis. Activation of FXR by bile acids results in reduced bile acid synthesis and enhances bile acid disposition ${ }^{28,29}$. We hypothesized that PTB should be prevented by restoring bile acid homeostasis through FXR activation. To test the hypothesis, pregnant mice were treated with $\mathrm{CCl}_{4}$ alone or a combination of $\mathrm{CCl}_{4}$ and a synthetic $\mathrm{FXR}$ agonist GW 4064. As shown in Fig. 8a, GW4064 significantly increased the gestation days from $17.9 \pm 0.59$ in mice treated with $\mathrm{CCl}_{4}$ $(n=9)$ to $19.1 \pm 0.76$ in mice treated with both $\mathrm{CCl}_{4}$ and GW4064 $(n=13)\left(p=9.6 \times 10^{-4}\right)$. The PTB rates were markedly decreased from $100 \%$ to $38.5 \%(p=0.04)$ and the rates of live birth were dramatically improved from $25.6 \pm 27.8 \%$ to $91.4 \pm$ $20.9 \%(p=0.0025)$. As expected, sTBAs were significantly reduced from $38.3 \pm 4.1$ to $23.8 \pm 5.5 \mu \mathrm{M}\left(p=2.4 \times 10^{-6}\right)$. Similar to sTBA, AST levels were significantly decreased from $122.1 \pm$ 22.8 to $43 \pm 15.3 \mathrm{U} \mathrm{L}^{-1}\left(p=3.7 \times 10^{-8}\right)$ and positively correlated with sTBA concentrations $(r=0.928, p<0.0001$; Fig. 8b). sTBA concentrations negatively correlated with the gestation days $(r=$ $-0.775, p=0.005)$ but not with the rates of live birth (Supplementary Fig. 8a). Serum AST levels negatively correlated with 
a

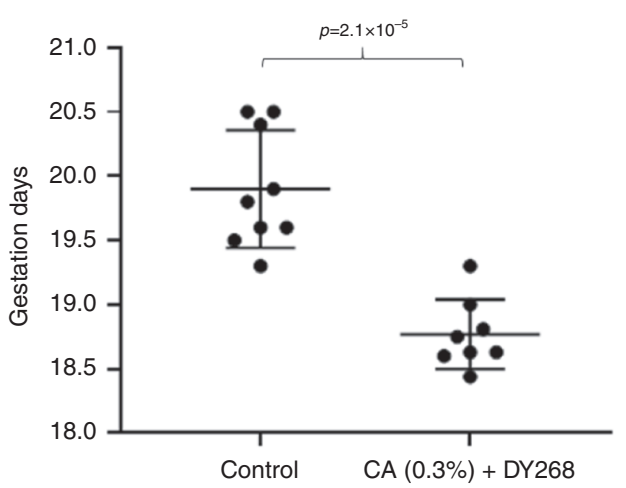

b

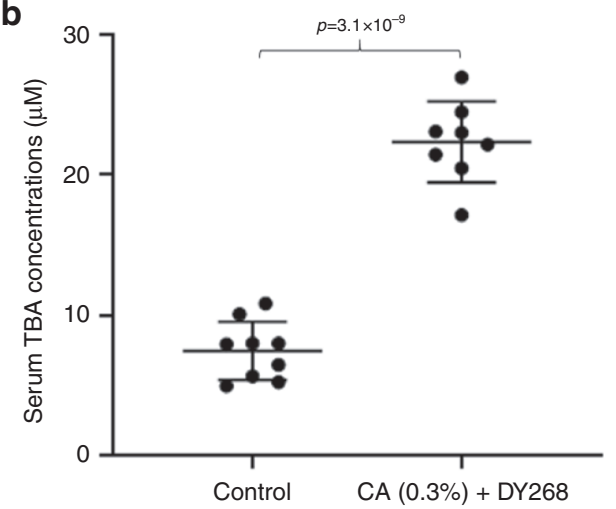

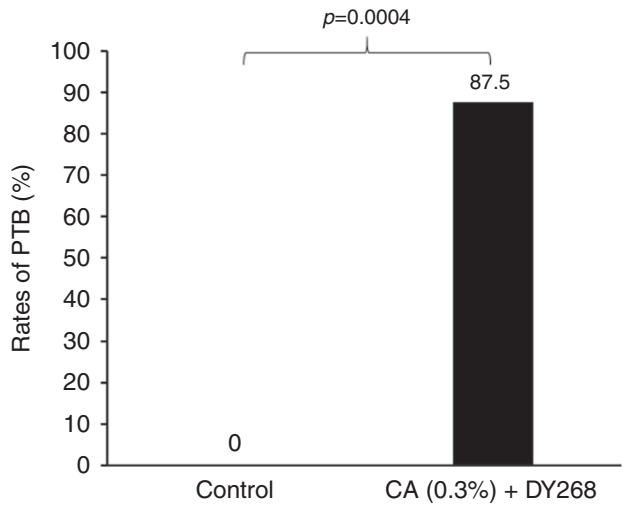

C

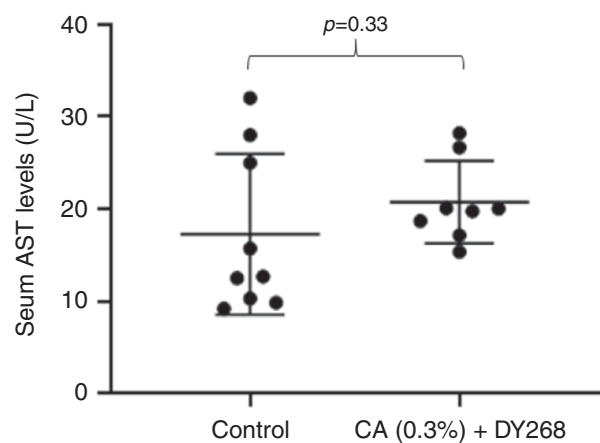

d

Mice 1

Mice 2
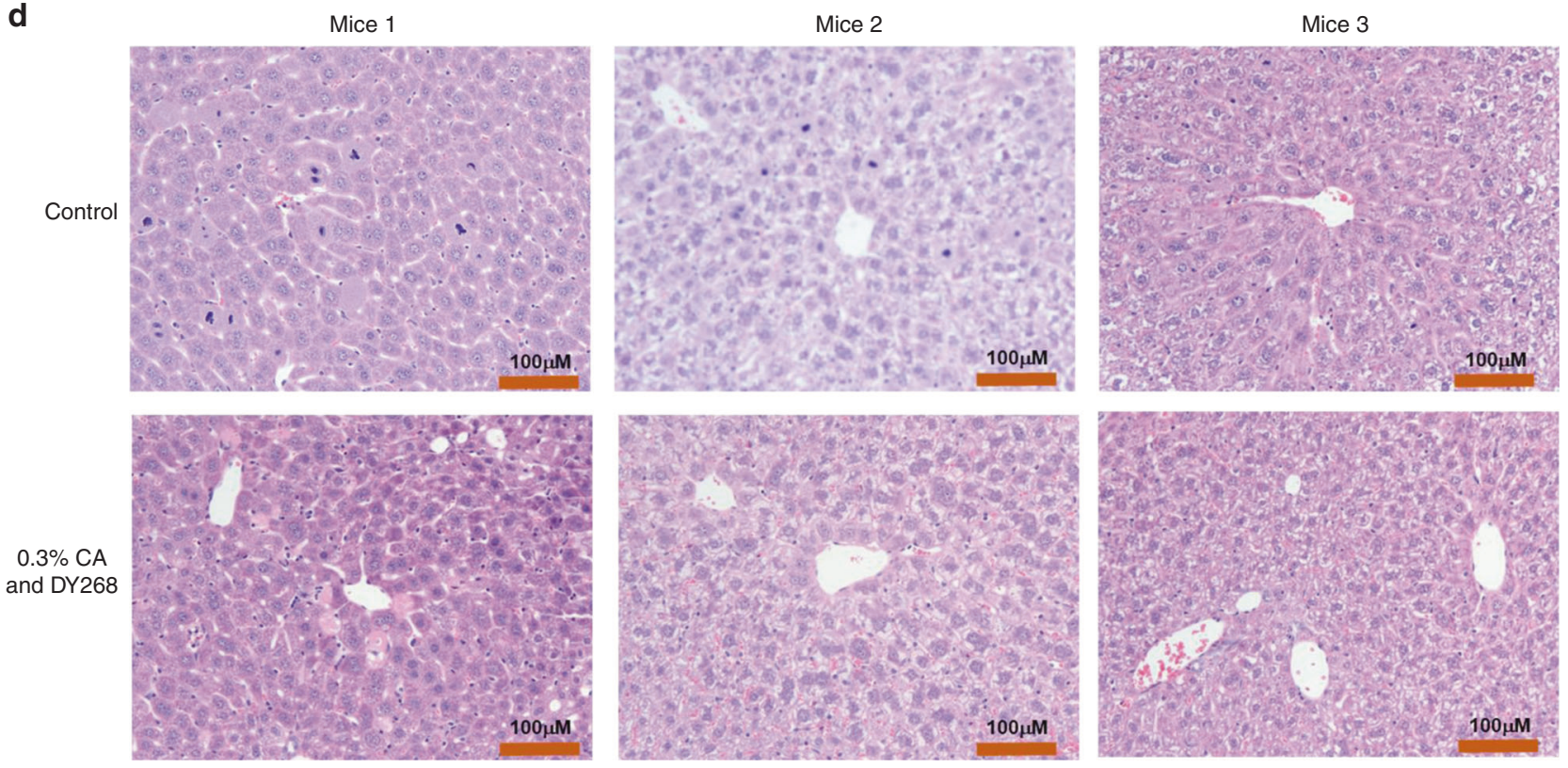

Fig. 7 Elevated bile acids, not liver injury, induced PTB. a The gestation days and PTB rates in control $(n=9)$ and mice treated with a combination of $0.3 \%$ CA and DY268 $(n=8)$. b The sTBA concentrations in the control $(n=9)$ and treatment group $(n=8)$. c The AST levels in control $(n=9)$ and mice treated with a combination of CA and DY268 $(n=8)$. d Representative hepatic histopathology of control and mice treated with CA and DY267. Consistent results were obtained from the control $(n=8)$ and treated mice $(n=8)$. Student's $t$ test for pairwise comparison (two-sided) and Chi-squared test for proportion comparisons were applied for statistical analysis. Source data are provided as a Source Data file.

both the gestation days $(r=-0.725, p=0.012)$ and the rates of live birth ( $r=-0.66, p=0.038$; Supplementary Fig. $8 \mathrm{~b})$. A direct correlation between the gestation days and the rates of live birth was also detected ( $r=0.648, p=0.043$; Supplementary Fig. 8c).

As the transporter responsible for the rate-limiting biliary secretion of bile acids in the enterohepatic circulation, the expression of bile salt export pump (Bsep) was significantly upregulated by GW4064 ( $p=0.0031$; Fig. 8c), indicating enhanced biliary bile acid disposition. On the other hand, cholesterol $7 \alpha$-hydroxylase (Cyp7a1), a rate-limiting enzyme for bile acid synthesis, was significantly downregulated $(p=0.0092)$, suggesting reduced bile acid synthesis. Consistently, the 
a
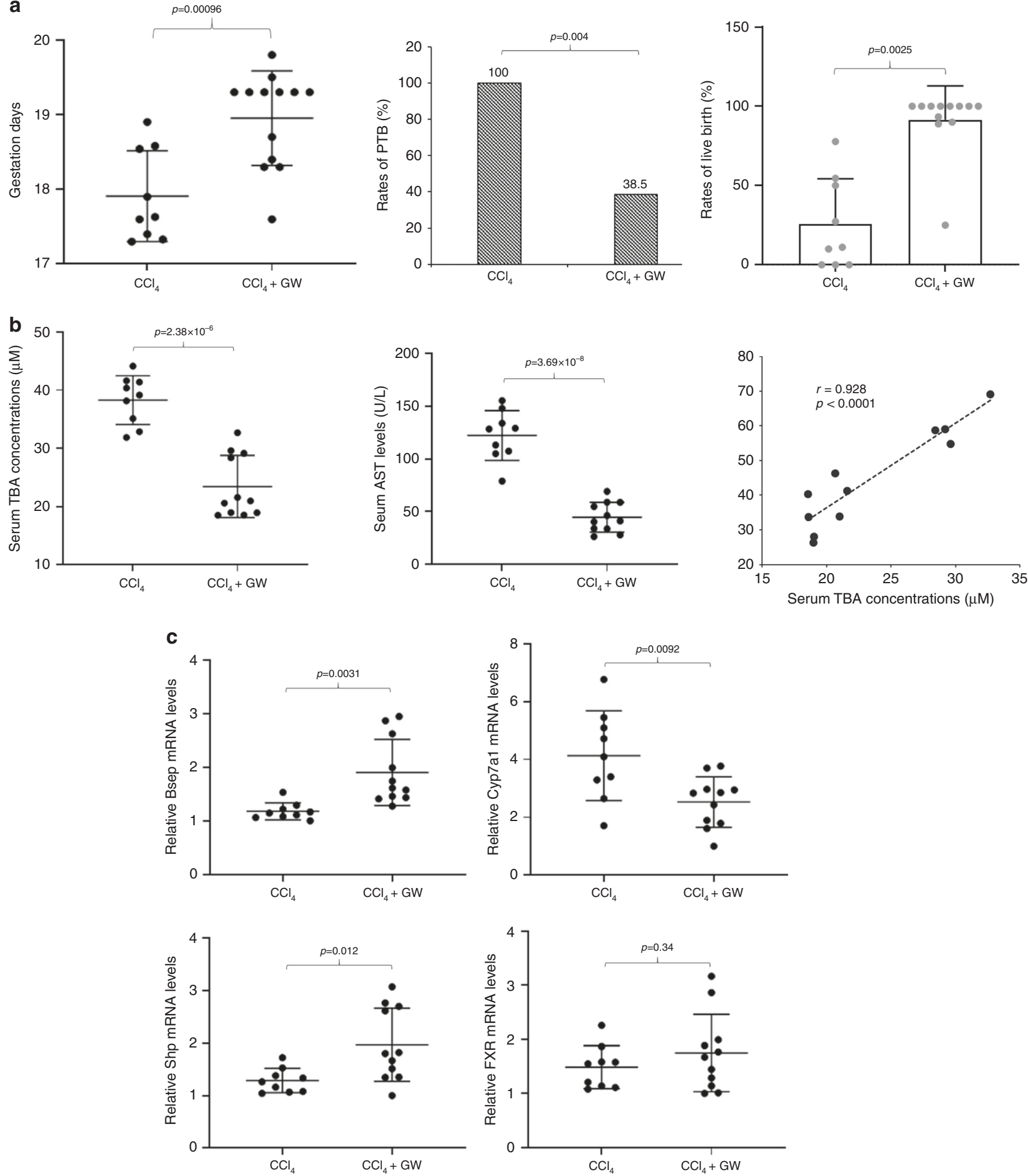

Fig. 8 Prevention of PTB by restoring bile acid homeostasis through FXR activation in pregnant mice. a The gestation days, PTB rates, and the rates of live birth in mice treated with $\mathrm{CCl}_{4}$ alone $\left(1.5 \mathrm{ml} \mathrm{kg}^{-1}\right)(n=9)$ or a combination of $\mathrm{CCl}_{4}\left(1.5 \mathrm{ml} \mathrm{kg}^{-1}\right)$ and FXR agonist GW4064 (50 mg kg-1) $(n=13)$. b The sTBA and AST levels in mice treated with $\mathrm{CCl}_{4}$ alone $(n=9)$ or a combination of $\mathrm{CCl}_{4}$ and $\mathrm{GW} 4064(n=11)$ and a positive correlation between sTBA and AST levels. c The relative expression levels of mRNAs for Bsep, Cyp7a1, Shp, and Fxr in mice treated with $\mathrm{CCl}_{4}(n=9)$ or a combination of CCl and GW4064 $(n=11)$. The means and standard errors of the group values were indicated by the long and short lines, respectively. Student's $t$ test for pairwise comparisons (two-sided) and Chi-squared test for proportion comparisons were applied for statistical analysis. Pearson correlation analysis was applied to determine the correlation coefficient and associated $p$ values. Source data are provided as a Source Data file. 
expression of small heterodimer partner $(S h p)$, a repressor for Cyp7a1 expression, was significantly increased $(p=0.012)$. On the other hand, GW4064 treatment had limited effects on Fxr expression ( $p=0.34$; Fig. $8 c$ ).

\section{Discussion}

The underlying mechanisms by which PTB is induced are complex and multi-factorial ${ }^{8,9}$. Bile acids are recently recognized as signaling molecules to regulate a variety of cellular and metabolic activities $^{14-16}$. In an early study to investigate the effects of bile acids on the fetus complications, direct intravenous infusion of $\mathrm{CA}$ into fetal lambs resulted in early delivery ${ }^{30}$. However, the cause of premature labor in the ewes with fetuses infused with CA was not evident. In this study, we found that sTBA concentrations in the pregnant women were directly correlated with the rates of PTB including SPTB and $\mathrm{P}$ PTB regardless of the characteristics of the pregnant women, such as maternal age and BMI or the etiologies of liver injuries, such as NAFLD and ICP. Our data are in line with the findings from previous reports that sTBA levels were correlated with the risks for PTB in ICP patients ${ }^{17-19}$. In this study, we further demonstrated that bile acid CA effectively and dose-dependently induced PTB in pregnant mice. The findings thus etiologically link elevated bile acids to PTB. Consistent with our finding, myometrium isolated from pregnant women with ICP was more sensitive to oxytocin than the myometrium from normal pregnant women, which may be due to the upregulation of oxytocin receptor expression in myometrium by bile acids ${ }^{31,32}$. Therefore, dysregulation of bile acids due to liver disorders or other pathological conditions is a previously unrecognized risk factor for PTB. sTBA concentrations may represent a potential predictive marker for PTB.

The liver is the organ responsible for nutritional and energy metabolism. As a physiological adaption, liver size is significantly enlarged during the late stage of pregnancy to meet the nutritional and metabolic demands of the mother and growing fetus $^{33-35}$. Under pathological conditions with dysregulated bile acids due to liver diseases or others, elevated bile acids may act as a message of compromised liver to communicate with and regulate other organs of the body. When the energy demands from the growing fetus and mother cannot be met by the compromised liver, termination of the pregnancy may become the option for the mother to survive. Therefore, early termination of pregnancy or PTB may be considered as a protective or adaptive mechanism for a pregnant woman to survive when liver function is compromised ${ }^{36,37}$. Consistently, similar to sTBA, serum AST, ALT, TBiL and GGT levels, which commonly serve as liver function or damage markers, directly correlated with the PTB rates in both human (Figs. 1d and 3c, Supplementary Figs. 1e, $\mathrm{f}$ and 2a, c) and mice (Supplementary Figs. 4a, 5a, 6a, and 8b). In addition, pregnant women with various liver disorders have increased risk for PTB ${ }^{17-19,38-45}$.

Advanced maternal age and being overweight/obese have been associated with increased risk for $\mathrm{PTB}^{20-27}$. However, the underlying mechanisms are unknown. In this study, we found that STBA concentrations were gradually elevated as the maternal age advanced (Fig. 2b) and BMI increased (Fig. 2d). More importantly, positive correlations between elevated sTBAs and increased PTB rates were detected in subjects with advanced maternal age and overweight or obese (Fig. 2b, d). Therefore, elevated circulating bile acids may directly contribute to the increased risk for PTB in those subjects. Consistent with our finding, previous clinical studies with non-pregnant subjects showed that serum bile acids were significantly elevated in obese subjects ${ }^{46-48}$. Currently, advanced age and overweight/obesity in reproductive women are on the rise, which may contribute to the current uptrend of PTB rate ${ }^{7}$. Modulating bile acid homeostasis may be of clinical significance for reducing PTB in age-advanced and overweight/obese subjects.

Among the liver disorders, NAFLD is considered the most common disease ${ }^{49-51}$. Indeed, NAFLD is the commonest cause for liver dysfunction in our study cohort, with $4.2 \%$ of the pregnant women being diagnosed with NAFLD. Similar to the subjects with ICP in this study and previous reports ${ }^{17-19}$, NAFLD patients also had increased PTB rates (Fig. 3a). The finding is consistent with a previous study reporting that the risk for PTB increased 2.5-fold in subjects with NAFLD ${ }^{42}$. However, the underlying mechanism is largely unknown. In this study, we found that sTBAs were also significantly elevated in NAFLD subjects (Fig. 3a) and thus may contribute to the induction of PTB in NAFLD subjects. Dysregulation of bile acids in subjects with NAFLD has long been recognized in non-pregnant subjects. A large body of evidence from clinical as well as preclinical studies demonstrate that sTBA concentrations were largely elevated in subjects with NAFLD ${ }^{52}$. Restoring bile acid homeostasis may represent a strategy to reduce the risk for PTB in pregnant women with NAFLD and ICP.

Elevated estrogens during late stages of pregnancy play an important role in the pathogenesis of $\mathrm{ICP}^{53-55}$. On the other hand, $\mathrm{CCl}_{4}$ has been widely used to induce various hepatic injuries in animals, including NAFLD, fibrosis, and non-alcoholic steatohepatitis ${ }^{56-58}$. In this study, we successfully reproduced PTB in pregnant mice with liver injuries induced by EE2 or $\mathrm{CCl}_{4}$. It was noted that high percentages of the newborns were dead in both EE2- and $\mathrm{CCl}_{4}$-induced PTB models. However, the underlying mechanisms may differ. In the $\mathrm{CCl}_{4}$ model, the rates of live birth negatively correlated with the gestation days (Supplementary Fig. 5b) with majority of dead births from the pregnant mice with extremely early gestation days (17.5 days). The finding is consistent with higher fetal mortality rates in pregnant women with extreme early PTB ${ }^{59,60}$. However, in the EE2 model, the rates of live birth did not directly correlate with the gestation days (Supplementary Fig. 4b), indicating that estrogen-induced cholestasis may have direct adverse effects on the fetuses. In patients with ICP, a high percentage of PTB is iatrogenic (73.5\%; Supplementary Fig. 3a, b), which are mainly due to fetal stresses and complications. In addition, increased stillbirths were also reported in pregnant women with $\mathrm{ICP}^{17-19}$. The high rates of dead births observed in the EE2 model may represent the sum of iPTB and stillbirth in pregnant women with ICP. It should be noted that the high dose of EE2 $\left(25 \mathrm{mg} \mathrm{kg}^{-1}\right)$ used in this study may also contribute to the high rates of dead birth. Taken together, EE2induced PTB may represent a good mouse model to study the pathogenesis of iPTB and stillbirth in pregnant women with ICP, while $\mathrm{CCl}_{4}$-induced PTB may represent a good mouse model to study PTB associated with liver disorders or dysregulation of bile acids other than ICP.

In this study, bile acid CA induced PTB as effectively as, if not more effectively than, EE2 and $\mathrm{CCl}_{4}$ treatments (Figs. 4a, 5a, 6a, and $7 \mathrm{a}$ ). Consistent with CA being a mild bile acid in inducing hepatotoxicity ${ }^{61}$, minimal or no liver injury was detected in mice treated with $0.5 \% \mathrm{CA}$ or a combination of $0.3 \% \mathrm{CA}$ and FXR antagonist DY268 as the AST levels were comparable to the control group (Figs. $6 c$ and $7 c$ ) and histopathology examination revealed no hepatic toxicity (Fig. $7 \mathrm{~d}$ ). The data thus demonstrate that it is the elevated bile acids, not liver injury itself, that induces PTB. Distinct from the EE2, $\mathrm{CCl}_{4}$ and other reported PTB mouse models ${ }^{62}$, which have low rates of live birth $(34.4 \pm 29.8$ for EE2 and $25.6 \pm 28.7$ for $\mathrm{CCl}_{4}$ model), majority of the newborns from CA-induced PTB were alive with a rate of live birth $85.0 \pm 10.6 \%$, $100 \%$, and $100 \%$ in mice treated with $1 \% \mathrm{CA}, 0.5 \% \mathrm{CA}$, and a combination of $0.3 \%$ CA and DY268, respectively. Therefore, 
CA-induced PTB represents a good model for studying the developmental consequences of the newborns. In addition, the findings also indicate that elevated bile acids are the trigger for inducing PTB while liver injuries induced by $\mathrm{EE} 2, \mathrm{CCl}_{4}$, or other factors may contribute to the low rates of live birth. Therefore, sTBA can potentially serve as a better marker for predicting PTB while other liver injury tests, such as AST, can potentially serve as better predictors for the health status of the fetus. Our findings indicated that reducing bile acids potentially prevent or delay PTB while promoting liver repairing or improving liver function may increase the survival rate of the newborns in pregnant women with liver disorders, including cholestasis. Considering the fact that some subjects had elevated bile acids but were protected from PTB, other factors such as individual variations in signaling pathways that mediate bile acid-induced PTB exist and remain to be identified.

Current interventions for preventing or delaying PTB did not achieve consistent improvement ${ }^{10,11}$. New mechanism-based interventions are in need. In this study, we demonstrated that restoring the bile acid homeostasis by FXR activation significantly reduced $\mathrm{CCl}_{4}$-induced PTB and dramatically improved the survival rates of the newborns. The finding holds a great promise for developing new therapies to prevent or delay PTB for pregnant women at high risk for PTB with dysregulated bile acids due to liver disorders or other conditions.

\section{Methods}

Study cohort. A prospective cohort was recruited at the Nantong Maternal and Child Health Hospital (NMCH) affiliated to Nantong University, China. The medical history and supporting clinical and laboratory information were collected at baseline. All pregnant women aged 18-50 years were screened. Exclusion criteria were described previously ${ }^{41}$. Data on maternal demographic characteristics were collected from questionnaires completed by women at the first antenatal visit ${ }^{41}$. A total of 44,906 pregnant women were screened during their first trimester of pregnancy at the $\mathrm{NMCH}$. Of these, 6242 were excluded owing to: multiple pregnancy in 1518 subjects, other infection in 2496 subjects, concurrent medical complications (diabetes, hypertension, or heart diseases) in 841 subjects, and spontaneous or induced abortion in 2787 subjects. Thereafter, 37,264 pregnant women were recruited in this study. Most of the enrolled subjects received at least three health examinations and were followed up until delivery. Five hundred and nine subjects were excluded because of lost to follow-up (292 subjects) or incomplete data (217 subjects). Finally, after exlusion, a total of 36,755 pregnant women were included in the study. We tried to include as many participants as possible and did not exclude participants if they fulfilled the inclusion criteria and follow-up. Self-selection bias was prevented by the blinding of investigators who collected the data and those who analyzed the data. Diagnoses of NAFLD and ICP were made according to relevant guidelines ${ }^{63,64}$. The primary outcome was PTB defined as earlier than 37 weeks of gestation. The PTB was further categorized into spontaneous (either following onset of contractions or spontaneously ruptured membranes) and iatrogenic (medically indicated labor due to maternal or fetal reasons) $\mathrm{PTB}^{65}$. The cohort study was performed according to the Declaration of Helsinki and approved by the Institutional Review Board of NMCH. Written informed consents were obtained from all participants. The study was retrospectively registered to the Research Registry (https://www.researchregistry.com/) with an identification number 5434 .

Chemicals and reagents. $\mathrm{CA}, \mathrm{CCl}_{4}, \mathrm{GW} 4064$, olive oil, and $\mathrm{EE} 2$ were purchased from Sigma-Aldrich. 1,2-Propanediol and corn oil were purchased from Acros Organics. Mouse TBA kits were purchased from Crystal Chem. Mouse AST Kit was purchased from Biovision.

\footnotetext{
Mouse models for PTB with liver injuries. Timed pregnancy of CD-1 mice was achieved by mating overnight. Next day when the male mice were removed is considered gestation day 0 . EE2 was administered subcutaneously once daily at a dose of $25 \mathrm{mg} \mathrm{kg}^{-1}$ in a solution of $80 \% 1,2$-propanediol and $0.15 \% \mathrm{NaCl}$ on gestation day 16.3. The treatment was continued until labor. Single injection of $\mathrm{CCl}_{4}$ was administered intraperitoneally at a dose of $1,1.5$, or $2 \mathrm{ml} \mathrm{kg}^{-1}$ in olive oil $(40 \% \mathrm{v} / \mathrm{v})$ on gestation day 16.3 . Upon initiation of the treatment, pregnant mice were closely monitored for labor. The rates of live birth were recorded. The mice were euthanized after the labor, and blood and tissues samples were collected for analyses. All the mouse studies were approved by the Institutional Animal Care and Use Committee of the University of Rhode Island. All mice were housed in ventilated cages in a 12-h light-dark cycle (starting at 6:00 a.m. and 6:00 p.m.), with
}

controlled room temperature $\left(20-22^{\circ} \mathrm{C}\right)$ and relative humidity (40\%). Mice had access to food and water ad libitum.

\section{Treatment of pregnant mice with bile acid CA and FXR antagonist DY268}

Treatment with $0.5 \%(\mathrm{w} / \mathrm{w})$ or $1 \% \mathrm{CA}$ in a regular chow diet was initiated at gestation day 15.3 and continued until labor. Treatment with a combination of $0.3 \%$ CA (in diet) and FXR antagonist DY268 $\left(20 \mathrm{mg} \mathrm{kg}^{-1}\right.$, oral gavage, twice a day) was also initiated at gestation day 15.3 and continued until labor. Pregnant mice were closely monitored for labor after initiation of the treatment. The rates of live birth were recorded. The mice were euthanized after the labor. Blood and tissues samples were collected for analyses. For the study with non-pregnant mice, mice were treated with $0.3 \%$ CA in diet or DY268 $\left(20 \mathrm{mg} \mathrm{kg}^{-1}\right.$, oral gavage, twice a day) for 3 days. At the end of the study, mice were euthanized, and blood samples were collected for sTBA and AST analyses.

\section{Treatment of pregnant mice with a combination of $\mathrm{CCl}_{4}$ and FXR agonist} GW4064. Pregnant mice were treated with FXR agonist GW4064 on gestation day 15.3 through oral gavage twice a day with a dose of $50 \mathrm{mg} \mathrm{kg}^{-1}$ in corn oil and the treatment continued till labor. Twenty-four hours after the first treatment of GW4064, the pregnant mice were treated with $\mathrm{CCl}_{4}$ at a dose of $1.5 \mathrm{ml} \mathrm{kg}^{-1}$ in $40 \%$ olive oil through intraperitoneal injection at gestation day 16.3. The mice were closely monitored for labor after $\mathrm{CCl}_{4}$ treatment. The gestation days and rates of live birth were recorded. The mice were euthanized after the labor. Blood and tissues samples were collected for analyses.

TaqMan quantitative real-time PCR. Total RNA was isolated with RNA-Bee regent (Tel-Test, Friendswood, TX). cDNA was synthesized with $1 \mu \mathrm{g}$ of total RNA and random primers in a total volume of $25 \mu$. The reactions were incubated initially at $25^{\circ} \mathrm{C}$ for $10 \mathrm{~min}$ and then at $50^{\circ} \mathrm{C}$ for $50 \mathrm{~min}$, followed by inactivation of the reaction at $70^{\circ} \mathrm{C}$ for $10 \mathrm{~min}$. The cDNAs were then diluted five times with water and subjected to real-time PCR using TaqMan Gene Expression Assay (Applied Biosystems, Foster City, CA). The real-time PCR was performed using TaqMan Universal PCR Master Mix (Applied Biosystems) in a total volume of 20 $\mu \mathrm{l}$ containing $10 \mu \mathrm{l}$ of universal PCR master mix, $1 \mu \mathrm{l}$ of gene-specific TaqMan assay mixture, and $5 \mu \mathrm{l}$ of cDNA templates. Cycling profile was $50^{\circ} \mathrm{C}$ for $2 \mathrm{~min}, 95$ ${ }^{\circ} \mathrm{C}$ for $10 \mathrm{~min}$, followed by $40 \mathrm{cycles}$ of $15 \mathrm{~s}$ at $95^{\circ} \mathrm{C}$ and $1 \mathrm{~min}$ at $60^{\circ} \mathrm{C}$, as recommended by the manufacturer. Amplification and quantification were done with the Applied Biosystems 7500 Real-Time PCR System. The TaqMan assay probes for Fxr (assay ID: Mm00436425_m1), Shp (assay ID: Mm00442278_m1), Cyp7a1 (assay ID: Mm00484150_m1), Bsep (assay ID: Mm00445168_m1), and glyceraldehyde 3-phosphate dehydrogenase (Gapdh) (assay ID: Mm99999915_g1) were purchased from Applied Biosystems. Transcript levels of Fxr, Shp, Cyp7a1, and Bsep were normalized against the GAPDH levels. Validated TaqMan PCR probes and master mixtures were obtained from Applied Biosystems.

Liver function tests. All recruited pregnant women had baseline liver function tests performed at the first antenatal visit at gestation weeks $12-14$, followed by at least one subsequent testing at gestation weeks 24-28. Additional testing was requested as clinically appropriate at gestation weeks $32-36$. Serum samples were collected from pregnant women after fasting for at least $8 \mathrm{~h}$. sTBA, AST, ALT, GGT, and TBiL levels were measured using an automatic biochemical analyzer (AU2700, Olympus, Japan). The highest values among the 2-3 tests for each individual subjects were used for the data analyses. Mouse sTBAs were quantified with the mouse TBA Kit from Crystal Chem. Mouse AST assays were carried out with the Mouse AST Kit from Biovision.

Histopathology. Fresh liver tissues were collected and fixed with $10 \%$ formalin immediately after euthanization of the mice. After fixation in $10 \%$ formalin solution for at least $24 \mathrm{~h}$, the liver tissue samples were delivered to the Molecular Pathology Core at Brown University for paraffin embedding, sectioning, hematoxylin and eosin staining, and histopathology examination by a pathologist. The samples were randomized and blinded when they were delivered to the core. After the histopathology report was provided to us, we decoded the samples and presented the data.

Statistics. Student's $t$ test was applied to pairwise comparison for normally distributed data. One-way analysis of variance was applied to analyze data with multiple groups, followed by Tukey post hoc test for multiple comparisons. Chisquared test was applied for proportion comparisons. Pearson correlation analysis was applied to determine the correlation coefficient $(r)$ and associated $p$ values. Poisson regression model was used to estimate the RR with $95 \%$ CI. A $p$ value of $\leq 0.05$ was considered statistically significant. IBM SPSS Statistics 25 , SAS, and Prism version 8 GraphPad software were used for statistical analyses.

Reporting summary. Further information on research design is available in the Nature Research Reporting Summary linked to this article. 


\section{Data availability}

All the relevant data that support the findings of this study are available from the corresponding author upon request. The source data underlying Figs. 1a-e, 2a-d, 3a-d, 4a, b, 5a, b, 6a-c, 7a-c, 8a-c and Supplementary Figs. 1a-k, 2a-d, 3a-d, 4a, b, 5a, b, 6a, b, $7 \mathrm{a}, \mathrm{b}, 8 \mathrm{a}-\mathrm{c}$ are provided as a Source Data file.

Received: 5 August 2019; Accepted: 3 April 2020;

Published online: 30 April 2020

\section{References}

1. March of Dimes, PMNCH, Save the Children, WHO. Born Too Soon: The Global Action Report on Preterm Birth (eds Howson, C. P., Kinney, M. V. \& Lawn, J. E.) (World Health Organization, Geneva, 2012).

2. McCormick, M. C. The contribution of low birth weight to infant mortality and childhood morbidity. N. Engl. J. Med. 312, 82-90 (1985).

3. Voltolini, C. et al. Understanding spontaneous preterm birth: from underlying mechanisms to predictive and preventive interventions. Reprod. Sci. 20 , 1274-1292 (2013).

4. Patel, R. M. Short- and long-term outcomes for extremely preterm infants. Am. J. Perinatol. 33, 318-328 (2016).

5. Markopoulou, P., Papanikolaou, E., Analytis, A., Zoumakis, E. \& Siahanidou, T. Preterm birth as a risk factor for metabolic syndrome and cardiovascular disease in adult life: a systematic review and meta-analysis. J. Pediatr. 210, 69. e5-80.e5 (2019).

6. World Health Organization. Key facts of preterm birth. https://www.who.int/ news-room/fact-sheets/detail/preterm-birth (2018). Updated 19 Feb 2018. Accessed 1 Apr 2019.

7. Centers for Disease Control and Prevention, National Center for Health Statistics. Percentages of birth born preterm by states $(2005,2014,2015,2016$ and 2017). https://www.cdc.gov/nchs/pressroom/sosmap/preterm_births/ preterm.htm (2019). Updated 15 Jan 2019. Accessed 2 Apr 2019.

8. Goldenberg, R. L., Culhane, J. F., Iams, J. D. \& Romero, R. Epidemiology and causes of preterm birth. Lancet 371, 75-84 (2008).

9. Frey, H. A. \& Klebanoff, M. A. The epidemiology, etiology, and costs of preterm birth. Semin. Fetal Neonatal Med. 21, 68-73 (2016).

10. Sykes, L. \& Bennett, P. R. Efficacy of progesterone for prevention of preterm birth. Best. Pract. Res. Clin. Obstet. Gynaecol. 52, 126-136 (2018).

11. Hanley, M. et al. A review of the literature. Obstet. Gynecol. Surv. 74, 50-55 (2019).

12. Gonzalez, F. J. Nuclear receptor control of enterohepatic circulation. Compr. Physiol. 2, 2811-2828 (2012).

13. Matsubara, T., Li, F. \& Gonzalez, F. J. FXR signaling in the enterohepatic system. Mol. Cell Endocrinol. 368, 17-29 (2013).

14. Shin, D. J. \& Wang, L. Bile acid-activated receptors: a review on Fxr and other nuclear receptors. Handb. Exp. Pharmacol. 256, 51-72 (2019).

15. Martinot, E. et al. Bile acids and their receptors. Mol. Asp. Med. 56, 2-9 (2017).

16. Jin, L. H., Fang, Z. P., Fan, M. J. \& Huang, W. D. Bile-ology: from bench to bedside. J. Zhejiang Univ. Sci. B 20, 414-427 (2019).

17. Geenes, V. et al. Association of severe intrahepatic cholestasis of pregnancy with adverse pregnancy outcomes: a prospective population-based casecontrol study. Hepatology 59, 1482-1491 (2014).

18. Glantz, A., Marschall, H. U. \& Mattsson, L. A. Intrahepatic cholestasis of pregnancy: Relationships between bile acid levels and fetal complication rates. Hepatology 40, 467-474 (2004)

19. Ovadia, C. et al. Association of adverse perinatal outcomes of intrahepatic cholestasis of pregnancy with biochemical markers: results of aggregate and individual patient data meta-analyses. Lancet 393, 899-909 (2019).

20. Zhang, $X$. et al. Changing trends of adverse pregnancy outcomes with maternal age in primipara with singleton birth: a join-point analysis of a multicenter historical cohort study in China in 2011-2012. Acta Obstet. Gynecol. Scand. 98, 997-1003 (2019).

21. Goisis, A., Remes, H., Barclay, K., Martikainen, P. \& Myrskylä, M. Advanced maternal age and the risk of low birth weight and preterm delivery: a withinfamily analysis using Finnish Population Registers. Am. J. Epidemiol. 186, 1219-1226 (2017).

22. Sohn, K. The trend in the relationship of advanced maternal age to preterm birth and low birthweight. Eur. J. Contracept. Reprod. Health Care 22, 363-368 (2017).

23. Fuchs, F., Monet, B., Ducruet, T., Chaillet, N. \& Audibert, F. Effect of maternal age on the risk of preterm birth: a large cohort study. PLOS ONE 13, e0191002 (2018).

24. Torloni, M. R. et al. Maternal BMI and preterm birth: a systematic review of the literature with meta-analysis. J. Matern Fetal Neonatal Med. 22, 957-970 (2009).
25. Vinturache, A., McKeating, A., Daly, N., Sheehan, S. \& Turner, M. Maternal body mass index and the prevalence of spontaneous and elective preterm deliveries in an Irish obstetric population: a retrospective cohort study. BMJ Open 7, e015258 (2017).

26. Kim, S. S., Mendola, P., Zhu, Y., Hwang, B. S. \& Grantz, K. L. Spontaneous and indicated preterm delivery risk is increased among overweight and obese women without prepregnancy chronic disease. BJOG 124, 1708-1716 (2017).

27. Cnattingius, S. et al. Maternal obesity and risk of preterm delivery. JAMA 309, 2362-2370 (2013).

28. Chiang, J. Y. Bile acid metabolism and signaling. Compr. Physiol. 3, 1191-1212 (2013).

29. Matsubara, T., Li, F. \& Gonzalez, F. J. FXR signaling in the enterohepatic system. Mol. Cell Endocrinol. 368, 17-29 (2013).

30. Campos, G. A., Guerra, F. A. \& Israel, E. J. Effects of cholic acid infusion in fetal lambs. Acta Obstet. Gynecol. Scand. 65, 23-26 (1986).

31. Israel, E. J., Guzman, M. L. \& Campos, G. A. Maximal response to oxytocin of the isolated myometrium from pregnant patients with intrahepatic cholestasis. Acta Obstet. Gynecol. Scand. 65, 581-582 (1986).

32. Germain, A. M., Kato, S., Carvajal, J. A., Valenzuela, G. J., Valdes, G. L. \& Glasinovic, J. C. Bile acids increase response and expression of human myometrial oxytocin receptor. Am. J. Obstet. Gynecol. 189, 577-582 (2003).

33. Hollister, A., Okubara, P., Watson, J. G. \& Chaykin, S. Reproduction in mice: liver enlargement in mice during pregnancy and lactation. Life Sci. 40, 11-18 (1987).

34. Milona, A. et al. The normal mechanisms of pregnancy-induced liver growth are not maintained in mice lacking the bile acid sensor Fxr. Am. J. Physiol. Gastrointest. Liver Physiol. 298, G151-G158 (2010).

35. Wang, F. et al. Telocytes in pregnancy-induced physiological liver growth. Cell. Physiol. Biochem. 36, 250-258 (2015).

36. Pike, I. L. Maternal stress and fetal responses: evolutionary perspectives on preterm delivery. Am. J. Hum. Biol. 17, 55-65 (2005)

37. Williams, T. C. \& Drake, A. J. Preterm birth in evolutionary context: a predictive adaptive response? Philos. Trans. R. Soc. Lond. B Biol. Sci. 374, 20180121 (2019).

38. Ludvigsson, J. F. et al. A population-based cohort study of pregnancy outcomes among women with primary sclerosing cholangitis. Clin. Gastroenterol. Hepatol. 12, 95.e1-100.e1 (2014).

39. Sirilert, S., Traisrisilp, K., Sirivatanapa, P. \& Tongsong, T. Pregnancy outcomes among chronic carriers of hepatitis B virus. Int. J. Gynaecol. Obstet. 126, 106-110 (2014).

40. Stokkeland, K. et al. Pregnancy outcome in more than 5000 births to women with viral hepatitis: a population-based cohort study in Sweden. Eur. J. Epidemiol. 32, 617-625 (2017).

41. Zhuang, X. et al. Liver dysfunction during pregnancy and its association of with preterm birth in China: a prospective cohort study. EBioMedicine 26, 152-156 (2017).

42. Hagström, H. et al. Adverse outcomes of pregnancy in women with nonalcoholic fatty liver disease. Liver Int. 36, 268-274 (2016).

43. Hagström, H. et al. Outcomes of pregnancy in mothers with cirrhosis: a national population-based cohort study of 1.3 million pregnancies. Hepatol. Commun. 2, 1299-1305 (2018).

44. Stokkeland, K. et al. Increased risk of preterm birth in women with autoimmune hepatitis - a nationwide cohort study. Liver Int. 36, 76-83 (2016).

45. Prodromidou, A. et al. Pregnancy outcomes after liver transplantation: a systematic review. Transpl. Proc. 51, 446-449 (2019).

46. Prinz, P. et al. Plasma bile acids show a positive correlation with body mass index and are negatively associated with cognitive restraint of eating in obese patients. Front. Neurosci. 9, 199 (2015).

47. Haeusler, R. A. et al. Increased bile acid synthesis and impaired bile acid transport in human obesity. J. Clin. Endocrinol. Metab. 101, 1935-1944 (2016).

48. Halmy, L., Fehér, T., Steczek, K. \& Farkas, A. High serum bile acid level in obesity: its decrease during and after total fasting. Acta Med. Hung. 43, 55-58 (1986).

49. Perumpail, B. J. et al. Clinical epidemiology and disease burden of nonalcoholic fatty liver disease. World J. Gastroenterol. 23, 8263-8276 (2017).

50. Arshad, T., Golabi, P., Paik, J., Mishra, A. \& Younossi, Z. M. Prevalence of nonalcoholic fatty liver disease in the female population. Hepatol. Commun. 3, 74-83 (2018)

51. Golabi, P. et al. Prevalence and outcomes of non-alcoholic fatty liver disease (NAFLD) among Asian American adults in the United States. Liver Int. 39, 748-757 (2019).

52. Zhang, X. \& Deng, R. in Nonalcoholic Fatty Liver Disease - An Update (ed. Gad, E. H.) 1-33 (IntechOpen Limited, London, 2018).

53. Leslie, K. K. et al. Estrogens in intrahepatic cholestasis of pregnancy. Obstet. Gynecol. 95, 372-376 (2000). 
54. Song, $\mathrm{X}$. et al. Transcriptional dynamics of bile salt export pump during pregnancy: mechanisms and implications in intrahepatic cholestasis of pregnancy. Hepatology 60, 1993-2007 (2014).

55. Abu-Hayyeh, S. et al. Intrahepatic cholestasis of pregnancy levels of sulfated progesterone metabolites inhibit farnesoid X receptor resulting in a cholestatic phenotype. Hepatology 57, 716-726 (2013)

56. Riordan, J. D. \& Nadeau, J. H. Modeling progressive non-alcoholic fatty liver disease in the laboratory mouse. Mamm. Genome 25, 473-486 (2014).

57. Domitrović, R., Jakovac, H., Tomac, J. \& Sain, I. Liver fibrosis in mice induced by carbon tetrachloride and its reversion by luteolin. Toxicol. Appl. Pharm. 241, 311-321 (2009).

58. Kubota, N. et al. A high-fat diet and multiple administration of carbon tetrachloride induces liver injury and pathological features associated with non-alcoholic steatohepatitis in mice. Clin. Exp. Pharmacol. Physiol. 40, 422-430 (2013).

59. MacDorman, M. F. \& Gregory, E. C. Fetal and perinatal mortality: United States, 2013. Natl. Vital Stat. Rep. 64, 1-24 (2015).

60. Li, Y. et al. The contribution of gestational age, area deprivation and mother's country of birth to ethnic variations in infant mortality in England and Wales: a national cohort study using routinely collected data. PLoS ONE 13, e0195146 (2018).

61. Attili, A. F., Angelico, M., Cantafora, A., Alvaro, D. \& Capocaccia, L. Bile acidinduced liver toxicity: relation to the hydrophobic-hydrophilic balance of bile acids. Med. Hypotheses 19, 57-69 (1986).

62. McCarthy, R. et al. Mouse models of preterm birth: suggested assessment and reporting guidelines. Biol. Reprod. 99, 922-937 (2018).

63. Chalasani, N. et al. The diagnosis and management of non-alcoholic fatty liver disease: practice guideline by the American Gastroenterological Association, American Association for the Study of Liver Diseases, and American College of Gastroenterology. Gastroenterology 142, 1592-1609 (2012).

64. Tran, T. T., Ahn, J. \& Reau, N. S. ACG Clinical Guideline: Liver Disease and Pregnancy. Am. J. Gastroenterol. 111, 176-194 (2016).

65. Di Renzo, G. C. et al. Preterm labor and birth management: recommendations from the European Association of Perinatal Medicine. J. Matern. Fetal Neonatal Med. 30, 2011-2030 (2017).

\section{Acknowledgements}

This work was partially funded by a research grant from AMAG Pharmaceuticals and was supported by the National Institutes of Health (NIH) Grant R01CA 213419. C.N. is partially supported by the pre-doctoral fellowship from American Foundation for Pharmaceutical Education. This work was also partially supported by a grant from Jiangsu provincial Department of Science and Technology, China (no. BE2015655). We thank all participants in this study cohort and the staff from the Department of Obstetrics, Nantong Maternal and Child Health Hospital affiliated to Nantong University for their work on patient's enrollment and follow-up.

\section{Author contributions}

R.D., G.Q., S.Y., A.-M.C., and S.F.H. designed the study. S.Y., A.-M.C., S.F.H., X.Z., C.N., Z.H., X.B., Q.C., W.A., and Y.C. performed the experiments. R.D., G.Q., S.Y., A.-M.C. and S.F.H. analyzed the data. R.D. and G.Q. wrote the manuscript. All authors reviewed and approved the manuscript.

\section{Competing interests}

The authors declare no competing interests.

\section{Additional information}

Supplementary information is available for this paper at https://doi.org/10.1038/s41467 020-15923-4.

Correspondence and requests for materials should be addressed to R.D.

Peer review information Nature Communications thanks the anonymous reviewer(s) for their contribution to the peer review of this work.

Reprints and permission information is available at http://www.nature.com/reprints

Publisher's note Springer Nature remains neutral with regard to jurisdictional claims in published maps and institutional affiliations.

(c) (i) Open Access This article is licensed under a Creative Commons Attribution 4.0 International License, which permits use, sharing, adaptation, distribution and reproduction in any medium or format, as long as you give appropriate credit to the original author(s) and the source, provide a link to the Creative Commons license, and indicate if changes were made. The images or other third party material in this article are included in the article's Creative Commons license, unless indicated otherwise in a credit line to the material. If material is not included in the article's Creative Commons license and your intended use is not permitted by statutory regulation or exceeds the permitted use, you will need to obtain permission directly from the copyright holder. To view a copy of this license, visit http://creativecommons.org/ licenses/by/4.0/.

(C) The Author(s) 2020 